\title{
Analysis of a Four-Dimensional Hyperchaotic System Described by the Caputo-Liouville Fractional Derivative
}

\author{
Ndolane Sene (iD \\ Laboratoire Lmdan, Département de Mathématiques de la Décision, Université Cheikh Anta Diop de Dakar, \\ Faculté des Sciences Economiques et Gestion, BP 5683 Dakar Fann, Senegal \\ Correspondence should be addressed to Ndolane Sene; ndolanesene@yahoo.fr
}

Received 5 September 2020; Revised 13 October 2020; Accepted 18 November 2020; Published 28 November 2020

Academic Editor: Mustafa Cagri Kutlu

Copyright (c) 2020 Ndolane Sene. This is an open access article distributed under the Creative Commons Attribution License, which permits unrestricted use, distribution, and reproduction in any medium, provided the original work is properly cited.

A new four-dimensional hyperchaotic financial model is introduced. The novelties come from the fractional-order derivative and the use of the quadric function $x^{4}$ in modeling accurately the financial market. The existence and uniqueness of its solutions have been investigated to justify the physical adequacy of the model and the numerical scheme proposed in the resolution. We offer a numerical scheme of the new four-dimensional fractional hyperchaotic financial model. We have used the Caputo-Liouville fractional derivative. The problems addressed in this paper have much importance to approach the interest rate, the investment demand, the price exponent, and the average profit margin. The validation of the chaotic, hyperchaotic, and periodic behaviors of the proposed model, the bifurcation diagrams, the Lyapunov exponents, and the stability analysis has been analyzed in detail. The proposed numerical scheme for the hyperchaotic financial model is destined to help the agents decide in the financial market. The solutions of the 4D fractional hyperchaotic financial model have been analyzed, interpreted theoretically, and represented graphically in different contexts. The present paper is mathematical modeling and is a new tool in economics and finance. We also confirm, as announced in the literature, there exist hyperchaotic systems in the fractional context, which admit one positive Lyapunov exponent.

\section{Introduction}

Many behaviors of the dynamical systems are deterministic. The systems' future behaviors follow the same evolutions and are explained by the initial conditions and the past of the systems. Chaos theory is one of the mathematical domain which studies these types of dynamical systems and has received many investigations $[1,2]$. Lorenz [2] was the first author to propose the chaotic system in three-dimensional space, namely, chaotic attractor. Lorenz's work can probably be considered as the beginning of this discipline. It is well known the chaotic systems are nonlinear dynamical systems and are sensitive to their initial conditions. That is, when the initial conditions of the considered system have small differences or changes, it becomes complicated to predict the behaviors of the system [1]. This field of mathematics is strongly in relation to the control theory. This reason explains the many investigations related to chaos control. In general, the controllers try to eliminate the chaotic behaviors using synchronization methods or other techniques. Many studies also focus on the stability analysis of the chaotic systems $[1,3]$. Chaotic behaviors are observed in many realworld problems, in fluid flows, in weather and climate [2], in the stock market, in road traffics, and others. Chaos theory has many applications, too, in anthropology, computer science, economic [1], biology, physics [1], meteorology, and others. After Lorenz's proposition, many other types of chaotic systems appear in the literature. Recent investigations focus on the chaotic and hyperchaotic systems in economics; we have the chaotic financial system with threedimensional space (see in $[4,5]$ ); we have the four-dimensional hyperchaotic financial model.

Chaotic and hyperchaotic systems have many applications in finance and economics. There exist many nonlinear dynamical systems in finance markets that use chaotic systems to predict the markets' behavior. In [1], Xin et al. 
presented the 3D chaotic financial model and introduced a new four-dimensional fractional chaotic financial system. The numerical investigation to approximate the financial model's solutions and the stability analysis have been proposed too in this paper. In [6], Chen et al. investigated the 4D hyperchaotic financial model using the Lyapunov direct method; the authors have found suitable control to stabilize the considered financial model. They also give the numerical simulation of their results. In [4], Gao and Ma investigated the complex dynamical behaviors of a finance system as the chaos and Hopf bifurcation. In [7], Yu et al. proposed a novel four-dimensional chaotic financial model based on the classical chaotic financial model with three dimension. The authors in [7] added the average profit margin to the classical financial model with three dimension to obtain a new chaotic model. They also presented the new model, the stability analysis, and provided the numerical simulation of their new model. In [8], Kumar et al. constructed a new finance model too, namely, the four-dimensional chaotic financial model and used the Lyapunov direct method to study the stability of the equilibrium points and also proposed the numerical simulations of the new model. For more investigations, like the works proposed by $\mathrm{He}$ in $[9,10]$, Yichen and others authors in $[11,12]$, Pham in $[13,14]$, and Shirkavand in [15], see also in $[3,5]$.

As previously observed, the stability and numerical simulations are the main interests in the three- and fourdimensional chaotic financial models. They are many methods for solving the fractional differential equations as the homotopy analysis, the homotopy perturbation, the domain decomposition, the numerical schemes as Adams-Bashforth numerical discretization, and others. In many contexts, the stability and the convergence of the previous cited methods are not trivial, and the effectiveness of the method can be discussed. For example, with homotopy methods, what is the good number of iterations to be considered under which we have good approximations for the solutions of the model. For the numerical schemes, including the numerical schemes of the fractional operators, the implementations of the methods are not easy, and the unconditional stability does not include the Lipschitz continuous of the functions constituting the model. Lipschitz continuous is known as indispensable for the existence of the solutions for a particular model. Many inconveniences exist in these previous cited methods. In this paper, we introduce a new hyperchaotic financial model; we propose a new procedure for getting the solutions of the four-dimensional hyperchaotic financial model by using implicit numerical discretization. Before investigating the solutions of the considered model, we propose qualitative properties. It is to justify the physical adequacy of the financial model. Our motivations are to introduce a new hyperchaotic financial model and introduce the fractional-order derivative [16-18] in modeling the financial equations. The fractional-order derivative well describes the memory effect. Thus, our second motivation is the use of the Caputo derivative into mathematical modeling. The main novelty in our study is the introduction of a numerical scheme to obtain the phases portraits of the considered system, which include the discretization of the Riemann-Liouville fractional integral and use the Lipschitz continuous conditions for the stability and the convergence of the numerical scheme. In other words, the existence of the model's solutions is sufficient for the stability and convergence of the used numerical scheme. It is essential to mention in our numerical discretization that we do not use the discretization of the fractional derivative but we have used the discretization of the fractional integral, which is more useful and uses the analytical solutions discreetly. Our novelty in terms of the financial model introduces the quadric function $x^{4}$ to measure the variations of the interest rate. We manipulate very sensitive chaos systems. Thus, the introduction of this new function in the model will generate many changes in the stability of the equilibrium points and influence the chaos generated by the variation of the model's parameters. The significant impact generated by the quadric function will be focused on this present paper. The bifurcation and the Lyapunov exponents in terms of the fractional derivative are also among the main novelties of this paper. This paper shows how to use the bifurcation and the Lyapunov exponents in the fractional context to analyze the chaos theory. In terms of the characterization of the chaos, we will provide the existence of two positive Lyapunov exponents that are sufficient for the hyperchaotic behaviors in the integer version, but in the context of fractional-order derivative, the numbers of positive Lyapunov exponents are not an adequate definition to characterize the hyperchaotic dynamics because there exist systems which are hyperchaotic with the bifurcation diagrams but admits one positive Lyapunov exponent. Therefore, fractional calculus must find theory on Lyapunov exponents to characterize chaotic behaviors and hyperchaotic behaviors. Our investigation will confirm the work on the Lyapunov exponent proposed by Danca in [19] who found hyperchaotic systems with one positive Lyapunov exponent. In the literature of the fractional calculus, the financial models, or chaotic systems are addressed in the papers [20-23]. For applications of the fractional derivatives in real-world problems, see in [17, 18, 24-31, 42-44].

In Section 2, we introduce the definitions and the tools necessary for our investigations. In Section 3, we present the $4 \mathrm{D}$ hyperchaotic financial model in the context of the fractional-order derivative and consider the memory effect. In Section 4, we focus on the qualitative properties of the proposed model. In Section 5, we describe the procedure of the solutions for the model. In Section 6, we represent the solutions graphically in different contexts. In Section 7, we analyze the characterizations of the chaos using the bifurcation diagrams and the Lyapunov exponents. In Section 8, we provide stability analysis in the context of fractional calculus. In Section 9, we give the future directions of research and final remarks.

\section{Basic Definitions and Lemmas}

This section is consecrated to the definitions of fractional derivatives and integrals. We will utilize them in our investigations. We work with fractional derivatives with singular kernels. In other words, we use the Caputo-Liouville 
fractional derivative, the Riemann-Liouville fractional derivative, and their associated generalizations. It is not hard to see when the order $\alpha=1$, we recover the classical integral. It proves the Liouville-Riemann integral is a generalization of the classical integral to the arbitrary noninteger order. Its associated derivative is called the Riemann-Liouville fractional derivative.

Definition 1 (see $[35,36])$. The Liouville-Riemann fractional integral is described as the following form for the function $x:[0,+\infty[\longrightarrow \mathbb{R}$ :

$$
\left(I^{\alpha} x\right)(t)=\frac{1}{\Gamma(\alpha)} \int_{0}^{t}(t-s)^{\alpha-1} x(s) \mathrm{d} s,
$$

where the function $\Gamma($.$) represents the Gamma Euler$ function and with the order $\alpha>0$.

Definition 2 (see $[35,36]$ ). The Liouville-Riemann fractional derivative of order $\alpha \in(0.1)$ is described as the following form for the function $x:[0,+\infty[\longrightarrow \mathbb{R}$ :

$$
D^{\alpha} x(t)=\frac{1}{\Gamma(1-\alpha)} \frac{d}{\mathrm{~d} t} \int_{0}^{t}(t-s)^{-\alpha} x(s) \mathrm{d} s,
$$

where the function $\Gamma($.$) represents the Gamma Euler$ function.

Another fractional derivative proposed in the literature is called the Caputo-Liouville fractional derivative due to the inconvenience of the Riemann-Liouville derivative.

Definition 3 (see $[35,36]$ ). The Caputo fractional derivative of order $\alpha \in(0.1)$ is described as the following form for the function $x:[0,+\infty[\longrightarrow \mathbb{R}:$

$$
D_{c}^{\alpha} x(t)=\frac{1}{\Gamma(1-\alpha)} \int_{0}^{t}(t-s)^{-\alpha} x^{\prime}(s) \mathrm{d} s,
$$

where the function $\Gamma($.$) represents the Gamma Euler$ function.

The generalization of two above fractional derivatives and integrals is represented in the following lines.

Definition 4 (see [28, 37-39]). The generalized Riemann-Liouville integral is described as the following form for the function $x:[0,+\infty[\longrightarrow \mathbb{R}$ :

$$
I^{\alpha, \rho} x(t)=\frac{1}{\Gamma(\alpha)} \int_{0}^{t}\left(\frac{t^{\rho}-s^{\rho}}{\rho}\right)^{\alpha-1} x(s) \frac{\mathrm{d} s}{s^{1-\rho}},
$$

with the orders $\alpha, \rho$ satisfying the relation $\alpha, \rho>0$, the function gamma is $\Gamma($.$) , and for all t>0$.

Definition 5 (see [28, 37-39]). The generalized Liouville-Riemann fractional derivative of order $\alpha \in(0.1)$ is described as the following form for the function $x:[0,+\infty[\longrightarrow \mathbb{R}:$

$$
D^{\alpha, \rho} x(t)=\frac{1}{\Gamma(1-\alpha)} \frac{\mathrm{d}}{\mathrm{d} t} \int_{0}^{t}\left(\frac{t^{\rho}-s^{\rho}}{\rho}\right)^{-\alpha} x(s) \frac{\mathrm{d} s}{s^{1-\rho}},
$$

with the orders $\alpha, \rho$ satisfying the relation $\alpha, \rho>0$, the function gamma is $\Gamma($.), and for all $t>0$.

Definition 6 (see $[26,28,40,41]$ ). The generalized Caputo fractional derivative of order $\alpha \in(0.1)$ is described as the following form for the function $x:[0,+\infty[\longrightarrow \mathbb{R}$ :

$$
D^{\alpha, \rho} x(t)=\frac{1}{\Gamma(1-\alpha)} \int_{0}^{t}\left(\frac{t^{\rho}-s^{\rho}}{\rho}\right)^{-\alpha} x^{\prime}(s) \mathrm{d} s,
$$

with the orders $\alpha, \rho$ satisfying the relation $\alpha, \rho>0$, the function gamma is $\Gamma($.$) , and for all t>0$.

We continue the rest of the paper with the Caputo-Liouville fractional derivatives. That is, we recall the Laplace transform of the Caputo-Liouville derivative; we have the relation

$$
\mathscr{L}\left\{\left(D_{c}^{\alpha} x\right)(t)\right\}=s^{\alpha} \mathscr{L}\{x(t)\}-s^{\alpha-k-1} x(0) .
$$

with the order $\alpha$ respecting the condition $\alpha \in(0.1)$.

\section{Fractional Four-Dimensional Hyperchaotic Financial Model}

In this section, we present the fractional model considered in our investigations. Yu, Cai, and $\mathrm{Li}$ proposed the integerorder version of the $4 \mathrm{D}$ hyperchaotic financial model in [7]. The fractional version of their model is described with the Caputo-Liouville derivative by the following equations:

$$
\begin{aligned}
& D_{c}^{\alpha} x=z+y x-a x+u, \\
& D_{c}^{\alpha} y=1-b y-x^{2}, \\
& D_{c}^{\alpha} z=-x-c z, \\
& D_{c}^{\alpha} u=-\mathrm{d} x y-k u .
\end{aligned}
$$

We make the following assumptions related to the initial conditions:

$$
\begin{aligned}
& x(0)=1, \\
& y(0)=2, \\
& z(0)=0.5, \\
& u(0)=0.5 .
\end{aligned}
$$

In equations (8)-(11), the variable $x$ represents the interest rate, the variable $y$ represents the investment demand, the variable $z$ represents the price exponent, and the variable $u$ is an additional variable that represents the average profit margin. The parameter $a$ denotes the saving rate, the parameter $b$ represents the per investment cost, the parameter $c$ indicates the elasticity of demands, and the parameter $k$ is the average profit margin influence. 
In our new modeling, we suppose, due to the external phenomena, the fluctuations of the interest rate into the financial market cannot be measured adequately by the quadratic function $x^{2}$ (in equation (9)) all times. Therefore, we consider more accurately quadric function $x^{4}$, which can correct all the errors in the measurement of the real data. Thus, the new four-dimensional hyperchaotic financial model can be modeled as the following form:

$$
\begin{aligned}
& D_{c}^{\alpha} x=z+y x-a x+u, \\
& D_{c}^{\alpha} y=1-b y-x^{4}, \\
& D_{c}^{\alpha} z=-x-c z, \\
& D_{c}^{\alpha} u=-d x y-k u .
\end{aligned}
$$

The strange actuator is obtained with the following values:

$$
\begin{aligned}
& a=0.5, \\
& b=0.2, \\
& c=1.2, \\
& d=0.2, \\
& k=0.15 .
\end{aligned}
$$

Our motivations for the use of the fractional-order derivative are to extend the four-dimensional hyperchaotic financial model described by integer-order derivative to the fractional-order derivative. First, the fractional-order derivative takes into account the memory effect; that is, the past behaviors of the model explain the next behaviors of the model. The second is to adapt the answer given to Leibniz's question to the financial model. Note that, it is proved in the literature that we can calculate the derivative of the function $\mathrm{d}^{n} f / \mathrm{d} t^{n}$ when $n$ is noninteger. In the literature, all the models, including the derivatives, are described by the integer-order derivatives; the question is now what will happen with these models when the order of the derivative is noninteger. It is also proved in the literature the fractional-order derivative is more realistic in modeling physical and economics models. For example, many diffusion processes as the subdiffusion, the ballistic diffusion process, the superdiffusion process, and the superdiffusion process which exist in real-world problems cannot be obtained with the integer derivative but with fractional-order derivatives. All these reasons have motivated us in this present works.

Note that the 3D financial chaotic model proposed in the literature is given by the following equation:

$$
\begin{aligned}
& \partial_{t} x=z+y x-a x \\
& \partial_{t} y=1-b y-x^{2} \\
& \partial_{t} z=-x-c z
\end{aligned}
$$

We can observe the average profit margin is added in the initial model equations (18)-(20) to obtain the 4D hyperchaotic financial model. Note that the average profit margin captures more perfectly the behaviors in the market [7] because it depends on the interest rate and the investment demand. We will analyze in detail the impact of the interest rate and the investment demand in the average profit margin. It is not hard to observe the $4 \mathrm{D}$ hyperchaotic financial model is another representation of the $3 \mathrm{D}$ chaotic financial equation because the average profit margin impacts the interest rate. In addition, the price exponent and the average profit margin are independent indirectly. The first objective of this paper is to prove the physical adequacy of the fractional model by establishing the solution of the fractional model described by equations (13)-(16) exist and is unique. The Banach fixed theorem will be used.

\section{Existence and Uniqueness of the Model}

In this section, we prove the fractional equation defined by equations (13)-(16) has at least one solution. The technique of proof uses the Banach fixed theorem procedure. This section is important for proving the physical adequacy of the fractional differential equations. In mathematical views, it is not important to study a model when the solution does not exist. The previous reasons are the motivations of this section.

We consider the first differential equation (13), and we suppose the function defined by

$$
H(x, t)=z+y x-a x+u .
$$

The function $H$ needs to be Lipschitz continuous. We have the following procedure of demonstration:

$$
\begin{aligned}
\left\|H\left(x_{1}, t\right)-H\left(x_{2}, t\right)\right\| & =\left\|z+y x_{1}-a x_{1}+u-z-y x_{2}+a x_{2}-u\right\| \\
& =\left\|y\left(x_{1}-x_{2}\right)-a\left(x_{1}-x_{2}\right)\right\| \\
& \leq\|y\|\left\|x_{1}-x_{2}\right\|+a\left\|x_{1}-x_{2}\right\| \\
& \leq(a+\|y\|)\left\|x_{1}-x_{2}\right\| .
\end{aligned}
$$

Under the assumption, the state variable $y$ is bounded, that is, $\|y\| \leq \epsilon_{1}$, and we get the condition of the Lipschitz continuous given by

$$
\left\|H\left(x_{1}, t\right)-H\left(x_{2}, t\right)\right\| \leq \epsilon\left\|x_{1}-x_{2}\right\|,
$$

with the Lipschitz constant expressed as the form $\epsilon=a+\epsilon_{1}$. The second step of the application of the Banach fixed theorem consists of constructing Picard's operator. It is clear that the solution of the fractional differential equation represented by equation (13) is given by the following expression:

$$
x(t)-x(0)=I^{\alpha} H(x, t)
$$

Based on the form of the solution, we define the following Picard's operator: 


$$
Z x(t)=x(0)+I^{\alpha} H(x, t) .
$$

Before using this expression, it is important to prove the operator $Z$ is well-bounded. We have the following procedure:

$$
\begin{aligned}
\| Z((t)-x(0) \| & =\left\|I^{\alpha} H(x, t)\right\|, \\
& \leq I^{\alpha}\|H(x, t)\| .
\end{aligned}
$$

From the assumption that the function $H$ is Lipschitz continuous, there exists $k$ such that $\|H(x, t)\| \leq k$ and equation (26) becomes

$$
\begin{aligned}
\| Z x(t) & -x(0)\left\|\leq I^{\alpha}\right\| H(x, t) \|, \\
& \leq k I^{\alpha}(1) \leq k \frac{1}{\Gamma(1+\alpha)} a^{\alpha},
\end{aligned}
$$

with the condition $t \leq a$. That is, the operator $Z$ is wellbounded. In other words, it is well definite, and now we should prove the operator $Z$ is also a contraction. We have the following reasoning:

$$
\begin{aligned}
\|Z u(t)-Z v(t)\| & \leq I^{\alpha}\|H(u, t)-H(v, t)\|, \\
& \leq\|H(u, t)-H(v, t)\| I^{\alpha}(1) .
\end{aligned}
$$

Using the Lipschitz continuous condition established in equation (23), we have the following relationship:

$$
\begin{aligned}
\|Z u(t)-Z v(t)\| & \leq I^{\alpha, \rho}\|H(u, t)-H(v, t)\|, \\
& \leq\|H(u, t)-H(v, t)\| I^{\alpha, \rho}(1), \\
& \leq \epsilon\|u(t)-v(t)\| I^{\alpha, \rho}(1), \\
& \leq \epsilon \frac{1}{\Gamma(1+\alpha)} a^{\alpha}\|u(t)-v(t)\| .
\end{aligned}
$$

That is, the operator $Z$ is a contraction when the following condition is held:

$$
\frac{1}{\Gamma(\alpha+1)} a^{\alpha} \leq \frac{1}{\epsilon} .
$$

We conclude that the solution of the fractional differential equation represented in equation (13) exists after application of the Banach fixed point theorem. After existence, we will try to show the uniqueness of the solution. We first consider two different solutions expressed as $x_{1}$ and $x_{2}$ for our considered equation (13). We have in particular the relations given by

$$
\begin{aligned}
& x_{1}(t)=x_{1}(0)+I^{\alpha} H\left(t, x_{1}\right), \\
& x_{2}(t)=x_{2}(0)+I^{\alpha} H\left(t, x_{1}\right) .
\end{aligned}
$$

We evaluate the difference between equation (31) and equation (32), and we obtain the relation defined by

$$
x_{1}(t)-x_{2}(t)=I^{\alpha}\left[H\left(t, x_{1}\right)-H\left(t, x_{2}\right)\right] .
$$

After the application of the Euclidean norm to equation (33), we obtain the equations

$$
\begin{aligned}
\left\|x_{1}-x_{2}\right\| & =\left\|I^{\alpha}\left[H\left(t, x_{1}\right)-H\left(t, x_{2}\right)\right]\right\|, \\
& \leq I^{\alpha}\left\|H\left(t, x_{1}\right)-H\left(t, x_{2}\right)\right\|, \\
& \leq\left\|H\left(t, x_{1}\right)-H\left(t, x_{2}\right)\right\| I^{\alpha}(1), \\
& \leq\left[\frac{a^{\alpha}}{\Gamma(\alpha+1)}\right] \epsilon\left\|x_{1}-x_{2}\right\| .
\end{aligned}
$$

We have the following relation after calculations:

$$
\left\|x_{1}-x_{2}\right\|\left[1-\left[\frac{a^{\alpha}}{\Gamma(\alpha+1)}\right] \epsilon\right] \leq 0,
$$

from which we get that $\left\|x_{1}-x_{2}\right\| \leq 0$. By definition, Euclidean norm satisfies the condition $\left\|x_{1}-x_{2}\right\| \geq 0$. We conclude that after combining the previous relationships, the following result:

$$
x_{1}=x_{2} .
$$

The unicity of the solution of equation (13) of our hyperchaotic model follows from equation (33). And then, we get the existence and the uniqueness of the solution of the first equation (13).

We repeat the previous reasoning by utilizing the second fractional differential equation (14), and we suppose a new function defined by

$$
D(y, t)=1-\text { by }-x^{4} .
$$

The function $D$ needs to be Lipschitz continuous. We have the following procedure of demonstration:

$$
\begin{aligned}
\left\|D\left(y_{1}, t\right)-D\left(y_{2}, t\right)\right\| & =\left\|1-b y_{1}-x^{4}-1+b y_{2}+x^{4}\right\|, \\
& =\left\|b\left(y_{1}-y_{2}\right)\right\|, \\
& \leq b\left\|y_{1}-y_{2}\right\| .
\end{aligned}
$$

We have the Lipschitzian continuous condition given by the following condition:

$$
\left\|D\left(y_{1}, t\right)-D\left(y_{2}, t\right)\right\| \leq b\left\|y_{1}-y_{2}\right\|,
$$

with the Lipschitz constant expressed as the form $b$. The second step of the application of the Banach fixed theorem consists of constructing Picard's operator. It is clear that the solution of the fractional differential equation represented by equation (14) is given by the following expression:

$$
y(t)-y_{0}=I^{\alpha} D(y, t) .
$$

Based on the form of the solution, we define the following Picard's operator:

$$
T y(t)=y(0)+I^{\alpha} D(y, t) .
$$

Before using this expression, it is important to prove the operator $T$ is well-bounded. We have the following procedure:

$$
\| T\left(y(t)-y(0)\|=\| I^{\alpha} D(y, t)\left\|, \leq I^{\alpha}\right\| D(y, t) \| .\right.
$$


Form the assumption the function $T$ is Lipschitz continuous, there exists $k$ such that $\|D(x, t)\| \leq k$, and equation (42) becomes

$$
\|T y(t)-y(0)\| \leq I^{\alpha}\|D(y, t)\|, \leq k I^{\alpha}(1) \leq k \frac{1}{\Gamma(\alpha+1)} a^{\alpha},
$$

with the condition $t \leq r$. That is, the operator $T$ is wellbounded. In other words, it is well definite, and now we should prove the operator $T$ is also a contraction. We have the following reasoning:

$$
\begin{aligned}
\|T u(t)-T v(t)\| & \leq I^{\alpha}\|D(u, t)-D(v, t)\|, \\
& \leq\|D(u, t)-D(v, t)\| I^{\alpha}(1) .
\end{aligned}
$$

Using the Lipschitz continuous condition established in equation (38), we have the following relationship:

$$
\begin{aligned}
\|T u(t)-T v(t)\| & \leq I^{\alpha}\|D(u, t)-D(v, t)\|, \\
& \leq\|D(u, t)-D(v, t)\| I^{\alpha}(1), \\
& \leq b\|u(t)-v(t)\| I^{\alpha}(1), \\
& \leq b \frac{1}{\Gamma(\alpha+1)} a^{\alpha}\|u(t)-v(t)\| .
\end{aligned}
$$

That is, the operator $Z$ is a contraction when the following condition is held:

$$
\frac{1}{\Gamma(\alpha+1)} a^{\alpha} \leq \frac{1}{b} .
$$

We conclude that the solution of the fractional differential equation represented in equation (14) exists after application of the Banach fixed point theorem. After existence, we will try to show the uniqueness of the solution. We first consider two different solutions expressed as $y_{1}$ and $y_{2}$ for our considered equation (14). We have in particular the relations given by

$$
\begin{aligned}
& y_{1}(t)=y_{1}(0)+I^{\alpha} D\left(t, y_{1}\right), \\
& y_{2}(t)=y_{2}(0)+I^{\alpha} D\left(t, y_{1}\right) .
\end{aligned}
$$

We evaluate the difference between equation (47) and equation (48), and we obtain the relation defined by

$$
y_{1}(t)-y_{2}(t)=I^{\alpha}\left[D\left(t, y_{1}\right)-D\left(t, y_{2}\right)\right] \text {. }
$$

After the application of the Euclidean norm to equation (49), we have the equations

$$
\begin{aligned}
\left\|y_{1}-y_{2}\right\| & =\left\|I^{\alpha}\left[D\left(t, y_{1}\right)-D\left(t, y_{2}\right)\right]\right\|, \\
& \leq I^{\alpha}\left\|D\left(t, y_{1}\right)-D\left(t, y_{2}\right)\right\|, \\
& \leq\left\|D\left(t, y_{1}\right)-D\left(t, y_{2}\right)\right\| I^{\alpha}(1), \\
& \leq\left[\frac{a^{\alpha}}{\Gamma(\alpha+1)}\right] b\left\|y_{1}-y_{2}\right\| .
\end{aligned}
$$

We obtain the following relation after calculations:

$$
\left\|y_{1}-y_{2}\right\|\left[1-\left[\frac{a^{\alpha}}{\Gamma(\alpha+1)}\right] b\right] \leq 0,
$$

from which we get that $\left\|y_{1}-y_{2}\right\| \leq 0$. By definition, Euclidean norm satisfies the condition $\left\|y_{1}-y_{2}\right\| \geq 0$. We conclude that, after combining the previous relationships, the following relationship:

$$
y_{1}=y_{2}
$$

The unicity of the solution of equation (14) of our hyperchaotic model follows from equation (52). And then, we get the existence and the uniqueness of the solution of the second equation in our model.

We continue with the third fractional differential equation (15), and we utilize the following function:

$$
A(z, t)=-x-c z \text {. }
$$

The function $A$ needs to be Lipschitz continuous. We have the following procedure of the proof:

$$
\begin{aligned}
\left\|A\left(z_{1}, t\right)-A\left(z_{2}, t\right)\right\| & =\left\|-x-c z_{1}+x+c z_{2}\right\|, \\
& =\left\|c\left(z_{1}-z_{2}\right)\right\|, \\
& \leq c\left\|z_{1}-z_{2}\right\| .
\end{aligned}
$$

We have the Lipschitzian continuous condition given by the following condition:

$$
\left\|A\left(z_{1}, t\right)-A\left(z_{2}, t\right)\right\| \leq c\left\|z_{1}-z_{2}\right\|,
$$

with the Lipschitz constant expressed as the form $c$. The second step of the application of the Banach theorem consists of constructing Picard's operator. It is clear that the solution of the fractional differential equation represented by equation (15) is given by the following expression:

$$
z(t)-z_{0}=I^{\alpha} A(z, t)
$$

Based on the form of the solution, we define the following Picard's operator:

$$
B z(t)=z(0)+I^{\alpha} A(z, t) .
$$

Before using this expression, it is important to prove the operator $T$ is well-bounded. We have the following procedure:

$$
\begin{aligned}
\| B(z(t)-z(0) \| & =\left\|I^{\alpha} A(z, t)\right\|, \\
& \leq I^{\alpha}\|A(z, t)\| .
\end{aligned}
$$

From the assumption the function $B$ is Lipschitz continuous, there exists $k$ such that $\|A(z, t)\| \leq k$ and equation (58) becomes

$$
\begin{aligned}
\| B z(t) & -z(0)\left\|\leq I^{\alpha, \rho}\right\| A(z, t) \|, \\
& \leq k I^{\alpha}(1) \leq k \frac{1}{\Gamma(\alpha+1)} a^{\alpha},
\end{aligned}
$$

with the condition $t \leq a$. That is, the operator $B$ is wellbounded. In other words, it is well definite, and now we 
should prove the operator $T$ is also a contraction. We have the following reasoning:

$$
\begin{aligned}
\|B u(t)-B v(t)\| & \leq I^{\alpha}\|A(u, t)-A(v, t)\|, \\
& \leq\|A(u, t)-A(v, t)\| I^{\alpha}(1) .
\end{aligned}
$$

Using the Lipschitz continuous condition established in equation (55), we have the following relationship:

$$
\begin{aligned}
\|B u(t)-B v(t)\| & \leq I^{\alpha}\|A(u, t)-A(v, t)\|, \\
& \leq\|A(u, t)-A(v, t)\| I^{\alpha}(1), \\
& \leq c\|u(t)-v(t)\| I^{\alpha}(1), \\
& \leq c \frac{1}{\Gamma(\alpha+1)} a^{\alpha}\|u(t)-v(t)\|,
\end{aligned}
$$

That is, the operator $B$ is a contraction when the following condition is held:

$$
\frac{1}{\Gamma(\alpha+1)} a^{\alpha} \leq \frac{1}{c} .
$$

We conclude that the solution of the fractional differential equation represented in equation (15) exists after application of the Banach fixed point theorem. Now, we will try to show the uniqueness of the solution. We first consider two different solutions expressed as $z_{1}$ and $z_{2}$ for our considered equation (15). We have in particular the relations given by

$$
\begin{aligned}
& z_{1}(t)=z_{1}(0)+I^{\alpha} A\left(t, z_{1}\right), \\
& z_{2}(t)=z_{2}(0)+I^{\alpha} A\left(t, z_{1}\right) .
\end{aligned}
$$

We evaluate the difference between equation (63) and equation (64), and we obtain the relation defined by

$$
z_{1}(t)-z_{2}(t)=I^{\alpha}\left[A\left(t, z_{1}\right)-A\left(t, z_{2}\right)\right] .
$$

After the application of the Euclidean norm to equation (65), we obtain the following equations:

$$
\begin{aligned}
\left\|z_{1}-z_{2}\right\| & =\left\|I^{\alpha}\left[A\left(t, z_{1}\right)-A\left(t, z_{2}\right)\right]\right\|, \\
& \leq I^{\alpha}\left\|A\left(t, z_{1}\right)-A\left(t, z_{2}\right)\right\|, \\
& \leq\left\|A\left(t, z_{1}\right)-A\left(t, z_{2}\right)\right\| I^{\alpha}(1), \\
& \leq\left[\frac{a^{\alpha}}{\Gamma(\alpha+1)}\right] c\left\|z_{1}-z_{2}\right\| .
\end{aligned}
$$

We obtain the following relation after calculations:

$$
\left\|z_{1}-z_{2}\right\|\left[1-\left[\frac{a^{\alpha}}{\Gamma(\alpha+1)}\right] c\right] \leq 0
$$

from which we get that $\left\|z_{1}-z_{2}\right\| \leq 0$. By definition, Euclidean norm satisfies the condition $\left\|z_{1}-z_{2}\right\| \geq 0$. We conclude that after combining the previous relationships,

$$
z_{1}=z_{2}
$$

The unicity of the solution of equation (15) of our hyperchaotic model follows from equation (68). And then, we get the existence and the uniqueness of the solution of the third equation in our considered model.

We finish with the fourth fractional differential equation (16), and we use the following function:

$$
\mathrm{Q}(u, t)=-\mathrm{d} x y-k u \text {. }
$$

The function $Q$ needs to be Lipschitz continuous. We have the following procedure of the proof:

$$
\begin{aligned}
\left\|Q\left(u_{1}, t\right)-Q\left(u_{2}, t\right)\right\| & =\left\|-\mathrm{d} x y-k u_{1}+\mathrm{d} x y+k u_{2}\right\|, \\
& =\left\|k\left(u_{1}-u_{2}\right)\right\|, \\
& \leq k\left\|u_{1}-u_{2}\right\| .
\end{aligned}
$$

We have the Lipschitz continuous condition given by the following condition:

$$
\left\|Q\left(u_{1}, t\right)-Q\left(u_{2}, t\right)\right\| \leq k\left\|u_{1}-u_{2}\right\|
$$

with the Lipschitz constant expressed as the form $c$. The second step of the application of the Banach fixed theorem consists of constructing Picard's operator. It is clear that the solution of the fractional differential equation represented by equation (16) is given by the following expression:

$$
u(t)-u_{0}=I^{\alpha} \mathrm{Q}(u, t)
$$

Based on the form of the solution, we define the following Picard's operator:

$$
K u(t)=u(0)+I^{\alpha} Q(u, t) .
$$

Before using this expression, it is important to prove the operator $K$ is well-bounded. We have the following procedure:

$$
\| K\left(u(t)-u(0)\|=\| I^{\alpha, \rho} Q(u, t)\left\|, \leq I^{\alpha}\right\| Q(u, t) \| .\right.
$$

From the assumption that the function $K$ is Lipschitz continuous, there exists $m$ such that $\|Q(u, t)\| \leq m$ and equation (74) becomes

$$
\begin{aligned}
\|K u(t)-u(0)\| & \leq I^{\alpha}\|Q(u, t)\|, \\
& \leq m I^{\alpha}(1) \leq m \frac{1}{\Gamma(\alpha+1)} a^{\alpha},
\end{aligned}
$$

with the condition $t \leq a$. That is, the operator $K$ is wellbounded. In other words, it is well definite, and now we should prove the operator $K$ is also a contraction. We have the following reasoning:

$$
\begin{aligned}
\|K v(t)-K w(t)\| & \leq I^{\alpha}\|Q(v, t)-Q(w, t)\|, \\
& \leq\|Q(v, t)-Q(w, t)\| I^{\alpha}(1) .
\end{aligned}
$$

Using the Lipschitz continuous condition established in equation (71), we have the following relationship: 


$$
\begin{aligned}
\|K v(t)-K w(t)\| & \leq I^{\alpha}\|Q(v, t)-Q(w, t)\|, \\
& \leq\|Q(v, t)-Q(w, t)\| I^{\alpha}(1), \\
& \leq k\|v(t)-w(t)\| I^{\alpha}(1), \\
& \leq k \frac{1}{\Gamma(\alpha+1)} a^{\alpha}\|v(t)-w(t)\| .
\end{aligned}
$$

That is, the operator $K$ is a contraction when the following condition is held:

$$
\frac{1}{\Gamma(\alpha+1)} a^{\alpha} \leq \frac{1}{k}
$$

We conclude that the solution of the fractional differential equation represented in equation (16) exists after application of the Banach fixed point theorem. After existence, we will try to show the uniqueness of the solution. We first consider two different solutions expressed as $u_{1}$ and $u_{2}$ for our considered equation (16). We have in particular the relations given by

$$
\begin{aligned}
& u_{1}(t)=u_{1}(0)+I^{\alpha} Q\left(t, u_{1}\right), \\
& u_{2}(t)=u_{2}(0)+I^{\alpha} Q\left(t, u_{1}\right) .
\end{aligned}
$$

We evaluate the difference between equation (79) and equation (80), and we obtain the relation defined by

$$
u_{1}(t)-u_{2}(t)=I^{\alpha}\left[Q\left(t, u_{1}\right)-Q\left(t, u_{2}\right)\right] .
$$

After the application of the Euclidean norm to equation (81), we obtain the equations

$$
\begin{aligned}
\left\|u_{1}-u_{2}\right\| & =\left\|I^{\alpha}\left[Q\left(t, u_{1}\right)-Q\left(t, u_{2}\right)\right]\right\|, \\
& \leq I^{\alpha}\left\|Q\left(t, u_{1}\right)-Q\left(t, u_{2}\right)\right\|, \\
& \leq\left\|Q\left(t, u_{1}\right)-Q\left(t, u_{2}\right)\right\| I^{\alpha}(1), \\
& \leq\left[\frac{a^{\alpha}}{\Gamma(\alpha+1)}\right] k\left\|u_{1}-u_{2}\right\| .
\end{aligned}
$$

We obtain the following relation after calculations:

$$
\left\|u_{1}-u_{2}\right\|\left[1-\left[\frac{a^{\alpha}}{\Gamma(\alpha+1)}\right] k\right] \leq 0,
$$

from which we get that $\left\|u_{1}-u_{2}\right\| \leq 0$. By definition of the euclidean norm satisfies the condition $\left\|u_{1}-u_{2}\right\| \geq 0$, we conclude that after combining the previous relationships,

$$
u_{1}=u_{2}
$$

The unicity of the solution of equation (16) of our hyperchaotic model follows from equation (84). And then, we get the existence and the uniqueness of the solution of the last equation in our considered model. We conclude that our financial hyperchaotic model has a solution, and this solution is unique. Finally, our general conclusion is our model is physically well defined. The research studies related to the solutions of our model are now well justified; we are sure the solution exists and is unique. The existence and uniqueness of the problem are very important in our context because it will justify the stability of the numerical schemes proposed in this paper. The solutions obtained with Picard's operator will play an important role in the discretization of the hyperchaotic model in context of fractional calculus.

\section{Solution Procedures of the Hyperchaotic Financial Model}

In this section, we address the numerical schemes of the equations in the proposed model. As we have mentioned early in the introduction, there are many methods for solving the differential equations: the Adams-Bashforth numerical method, the homotopy method, the Fourier methods, the Laplace transform method, and others. All the previous methods cannot be applied in all problems; for example, the Laplace transform application is not all-time possible due to the nonlinearities of some equations. With the homotopy method, the number of iterations after the method converges is the literature's main problem. Many of them consider between three or five iterations, but it do not ensures the convergence of the method. The Fourier transform has limitations because all functions have not their Fourier transforms. All the numerical schemes as the implicit or explicit numerical scheme can be used. Many numerical schemes are utilized to obtain the approximate solutions, but the existence and the uniqueness of the solutions of the models are not proved. In case the condition of existence is provided, the unconditional stability and the convergence of the methods are not trivial, and the applied methods to prove them can fail. Some of the considered numerical methods like homotopy can diverge because the number of iteration considered is small. Regarding the previous problem, we apply a numerical scheme to take into account the fractional integral, the analytical solution, and where the stability and the convergence can be obtained using the existence and the uniqueness of the solution of the proposed model. Our numerical scheme is similar to Adams Basfoth procedure in the context of integer order derivative. In our context, we use a similar procedure, but the difference is the discretization of the fractional integral instead of the classical integral. The numerical scheme comes from the Garrappa paper [42]. The innovation of this section is the use of the analytical solution and the numerical approximation of the Riemann-Liouville fractional integral. The present numerical scheme has many advantages regarding the classical Adams-Bashforth method and homotopy. The first advantage is with our method; we begin with the exact analytical solution of the model. The second advantage is the stability analysis and convergence. The convergence of our numerical scheme is ensured by the Lipschitz continuous of the model's drift functions, which are used to prove the existence and uniqueness. The third advantage is the use of the numerical schemes of the fractional integral, which is more useful than the numerical schemes of the fractional derivatives. The utilization of the Newton method to study the unconditional stability of the implicit or explicit numerical schemes is a severe inconvenience of classical numerical schemes; with our numerical scheme, the verification of the Lipschitz continuous solves this problem. Based on the 
fractional Riemann-Liouville integral and the fact the solution exists, the exact analytical solutions of the $4 \mathrm{D}$ hyperchaotic financial model (13)-(16) is represented by the following forms:

$$
\begin{aligned}
& x(t)=x(0)+I^{\alpha} H(x, t), \\
& y(t)=y(0)+I^{\alpha} D(y, t), \\
& z(t)=z(0)+I^{\alpha} A(z, t), \\
& u(t)=u(0)+I^{\alpha} Q(u, t) .
\end{aligned}
$$

In the context of the Adams Basford method, the order $\alpha$ is 1 . In our context, the order $\alpha$ is noninteger and belongs into the interval $(0,1)$. In difference with classical approximation proposed by Adams and Basford for the integral part, we discretize the integral part using the numerical approximation of the fractional integral. Now, we evaluate equations (85)-(88) at the point $\left(t_{n}\right)$, and we obtain the following equations with Riemann-Liouville fractional integral:

$$
\begin{gathered}
x\left(t_{n}\right)=x(0)+I^{\alpha} H\left(x, t_{n}\right), \\
y\left(t_{n}\right)=y(0)+I^{\alpha} D\left(y, t_{n}\right), \\
z\left(t_{n}\right)=z(0)+I^{\alpha} A\left(z, t_{n}\right), \\
u(t)=u(0)+I^{\alpha} Q\left(u, t_{n}\right) .
\end{gathered}
$$

Using the explicit form of the Riemann-Liouville fractional integral, we get the following equations:

$$
\begin{aligned}
& x\left(t_{n}\right)=x(0)+\frac{1}{\Gamma(\alpha)} \sum_{j=0}^{n} \int_{t_{j}}^{t_{j+1}}\left(t_{n}-s\right)^{\alpha-1} H(x(s), s), \\
& y\left(t_{n}\right)=y(0)+\frac{1}{\Gamma(\alpha)} \sum_{j=0}^{n-1} \int_{t_{j}}^{t_{j+1}}\left(t_{n}-s\right)^{\alpha-1} D(y(s), s), \\
& z\left(t_{n}\right)=z(0)+\frac{1}{\Gamma(\alpha)} \sum_{j=0}^{n-1} \int_{t_{j}}^{t_{j+1}}\left(t_{n}-s\right)^{\alpha-1} A(z(s), s), \\
& u\left(t_{n}\right)=u(0)+\frac{1}{\Gamma(\alpha)} \sum_{j=0}^{n-1} \int_{t_{j}}^{t_{j+1}}\left(t_{n}-s\right)^{\alpha-1} Q(z(s), s) .
\end{aligned}
$$

Let the grid $t_{n}=n h$, where $h$ denotes a constant step size, and the schemes of the fractional integral parts are given by

$$
\begin{aligned}
& I^{\alpha} H\left(t_{n}, x\right)=h^{\alpha} \sum_{j=1}^{n} \mu_{n-j} H\left(t_{j}, z_{j}\right), \\
& I^{\alpha} D\left(t_{n}, y\right)=h^{\alpha} \sum_{j=1}^{n} \mu_{n-j} D\left(t_{j}, z_{j}\right), \\
& I^{\alpha} A\left(t_{n}, z\right)=h^{\alpha} \sum_{j=1}^{n} \mu_{n-j} A\left(t_{j}, z_{j}\right),
\end{aligned}
$$

$I^{\alpha} Q\left(t_{n}, z\right)=h^{\alpha} \sum_{j=1}^{n} \mu_{n-j} Q\left(t_{j}, z_{j}\right)$,

where the parameter is given by $\mu_{n-j}=\left((n-j+1)^{\alpha}-(n-j)^{\alpha}\right) /(1 / \Gamma(1+\alpha))$. We use the first-order interpolant polynomial of the functions $H, D, A$, and $Q$ which are in the following forms:

$$
\begin{aligned}
H(\tau, x(\tau))= & H\left(t_{j+1}, z_{j+1}\right)+\frac{\tau-t_{j+1}}{h}\left[H\left(t_{j+1}, z_{j+1}\right)\right. \\
& \left.-H\left(t_{j}, z_{j}\right)\right], \\
D(\tau, y(\tau))= & D\left(t_{j+1}, z_{j+1}\right)+\frac{\tau-t_{j+1}}{h}\left[D\left(t_{j+1}, z_{j+1}\right)\right. \\
& \left.-D\left(t_{j}, z_{j}\right)\right], \\
A(\tau, z(\tau))= & A\left(t_{j+1}, z_{j+1}\right)+\frac{\tau-t_{j+1}}{h}\left[A\left(t_{j+1}, z_{j+1}\right)\right. \\
& \left.-A\left(t_{j}, z_{j}\right)\right], \\
Q(\tau, z(\tau))= & Q\left(t_{j+1}, z_{j+1}\right)+\frac{\tau-t_{j+1}}{h}\left[Q\left(t_{j+1}, z_{j+1}\right)\right. \\
& \left.-Q\left(t_{j}, z_{j}\right)\right] .
\end{aligned}
$$

We replace equations (95)-(98) into equations (91)-(94), and the numerical discretization of the fractional integral is given by the following expressions:

$$
\begin{aligned}
I^{\alpha} H\left(t_{n}, x\right) & =h^{\alpha}\left[\bar{\mu}_{n}^{(\alpha)} H(0)+\sum_{j=1}^{n} \mu_{n-j}^{(\alpha)} H\left(t_{j}, x_{j}\right)\right], \\
I^{\alpha} D i\left(t_{n}, y\right) & =h^{\alpha}\left[\bar{\mu}_{n}^{(\alpha)} D(0)+\sum_{j=1}^{n} \mu_{n-j}^{(\alpha)} D\left(t_{j}, y_{j}\right)\right], \\
I^{\alpha} A\left(t_{n}, z\right) & =h^{\alpha}\left[\bar{\mu}_{n}^{(\alpha)} A(0)+\sum_{j=1}^{n} \mu_{n-j}^{(\alpha)} A\left(t_{j}, z_{j}\right)\right], \\
I^{\alpha} Q\left(t_{n}, z\right) & =h^{\alpha}\left[\bar{\mu}_{n}^{(\alpha)} Q(0)+\sum_{j=1}^{n} \mu_{n-j}^{(\alpha)} Q\left(t_{j}, z_{j}\right)\right] .
\end{aligned}
$$

The implicit schemes of equations (13)-(16) are given the following discretizations:

$$
\begin{aligned}
& x\left(t_{n}\right)=x(0)+h^{\alpha}\left[\bar{\mu}_{n}^{(\alpha)} \varphi(0)+\sum_{j=0}^{n} \mu_{n-j}^{(\alpha)} H\left(x_{j}, t_{j}\right)\right], \\
& y\left(t_{n}\right)=y(0)+h^{\alpha}\left[\bar{\mu}_{n}^{(\alpha)} \varphi(0)+\sum_{j=0}^{n} \mu_{n-j}^{(\alpha)} D\left(y_{j}, t_{j}\right)\right], \\
& z\left(t_{n}\right)=z(0)+h^{\alpha}\left[\bar{\mu}_{n}^{(\alpha)} \varphi(0)+\sum_{j=0}^{n} \mu_{n-j}^{(\alpha)} A\left(z_{j}, t_{j}\right)\right], \\
& u\left(t_{n}\right)=u(0)+h^{\alpha}\left[\bar{\mu}_{n}^{(\alpha)} \varphi(0)+\sum_{j=0}^{n} \mu_{n-j}^{(\alpha)} Q\left(u_{j}, t_{j}\right)\right],
\end{aligned}
$$

where the parameters used above are represented as follows: 


$$
\bar{\mu}_{n}^{(\alpha)}=\frac{(n-1)^{\alpha}-n^{\alpha}(n-\alpha-1)}{\Gamma(2+\alpha)},
$$

and furthermore, for $n=1,2, \ldots$,

$$
\begin{aligned}
& \mu_{0}^{(\alpha)}=\frac{1}{\Gamma(2+\alpha)}, \\
& \mu_{n}^{(\alpha)}=\frac{(n-1)^{\alpha+1}-2 n^{\alpha+1}+(n+1)^{\alpha+1}}{\Gamma(2+\alpha)} .
\end{aligned}
$$

We consider the approximate solutions $x\left(t_{n}\right), y\left(t_{n}\right), z\left(t_{n}\right)$, and $u\left(t_{n}\right)$ of equations (13)-(16). We assume $x_{n}, y_{n}, z_{n}$, and $u_{n}$ the exact solutions of equations (13)-(16). Then, the residual functions for the implicit discretization are given by the functions:

$$
\begin{aligned}
& \left|x\left(t_{n}\right)-x_{n}\right|=\mathcal{O}\left(h^{\min \{\alpha+1,2\}}\right), \\
& \left|y\left(t_{n}\right)-y_{n}\right|=\mathcal{O}\left(h^{\min \{\alpha+1,2\}}\right), \\
& \left|z\left(t_{n}\right)-z_{n}\right|=\mathcal{O}\left(h^{\min \{\alpha+1,2\}}\right), \\
& \left|u\left(t_{n}\right)-u_{n}\right|=\mathcal{O}\left(h^{\min \{\alpha+1,2\}}\right) .
\end{aligned}
$$

The convergence of the implicit schemes of equations (13)-(16) is obtained when the parameter $h$ converges to 0 . The stability of our numerical discretizations in this paper is obtained when the functions $H, D, A$, and $Q$ are the Lipschitz continuous, which are proved in the section related to the existence and the uniqueness. The following equations represent the numerical discretizations of the functions $H, D, A$, and $Q$ :

$$
\begin{aligned}
& H\left(x_{j}, t_{j}\right)=z^{j}+y^{j} x^{j}-a x^{j}, \\
& D\left(y_{j}, t_{j}\right)=1-b y^{j}-\left(x^{j}\right)^{4}, \\
& A\left(z_{j}, t_{j}\right)=-x^{j}-c z^{j}, \\
& Q\left(u_{j}, t_{j}\right)=-d x^{j} y^{j}-k u^{j} .
\end{aligned}
$$

\section{Behavior of the Solutions with Fractional Orders}

In this section, we represent graphically the interest rate, the investment demand, the price exponent, and the average profit margin obtained after solving the fractional $4 \mathrm{D}$ hyperchaotic financial model (13)-(16) with our numerical scheme described in the previous section. We analyze and also interpret the different behaviors. In this section, we make the following assumptions related to the different parameters: the saving rate $a=0.5$, the per investment cost $b=0.2$, the elasticity of demands $c=1.2, d=0.2$, and the average profit margin influence $k=0.15$. The initial conditions are given, respectively, as follows:

$$
\begin{aligned}
& x(0)=0.5, \\
& y(0)=0.5, \\
& z(0)=0.5, \\
& u(0)=0.5 .
\end{aligned}
$$

To see the fractional order's influence in the hyperchaotic dynamics, we consider four values of the Caputo fractional-order derivative: $\alpha=0.95, \alpha=0.90, \alpha=0.88$, and $\alpha=0.45$. In our first case, we consider the order $\alpha=0.95$. In Figures 1-4, we consider the interest rate, the investment demand, and the price exponent and with order $\alpha=0.95$, in different angles.

In Figures 5-7, we consider the interest rate, the investment demand, and the average profit margin and with order $\alpha=0.95$, in different context.

In our third case, in terms of comparison with the previous considered orders, we consider the order $\alpha=0.90$. In Figures 8-11, we consider the interest rate, the investment demand, and the price exponent and with order $\alpha=0.90$, in different angles.

In Figures 12-14, we consider the interest rate, the investment demand, and the average profit margin and with order $\alpha=0.90$, in different context.

In our third case, in terms of comparison with the previous considered order, we consider the order $\alpha=0.88$. In Figures 15-18, we consider the interest rate, the investment demand, and the price exponent and with order $\alpha=0.88$, in different angles.

In Figures 19-21, we consider the interest rate, the investment demand, and the average profit margin and with order $\alpha=0.88$, in different context.

In our last case, in terms of comparison with the previous considered orders, we consider the order $\alpha=0.45$. In Figures 22-25, we consider the interest rate, the investment demand, and the price exponent and with order $\alpha=0.45$, in different angles.

In Figures 26 and 27, we consider the interest rate, the investment demand, and the average profit margin and with order $\alpha=0.45$, in different context.

We notice the fractional-order derivative has a significant impact on the hyperchaotic behaviors of the considered model. We observe when the order $\alpha$ of the fractional derivative is into the interval $(0.85,1)$, we detect hyperchaotic behaviors. The hyperchaotic behaviors are immediately removed when the order approaches $\alpha=0.45$ as we can observe in the previous figures. With the above figures, we note strange actuators when the order is small and converge to $\alpha=0.90$ that means the agents will have many difficulties to predict the values of the interest rate, the investment demand, the price exponent, and the average profit margin in the financial market. In conclusion, the hyperchaotic systems are very sensitive to the order of fractional derivatives.

\section{Bifurcation and Lyapunov Exponent}

In this section, we have a new model; it is essential to analyze the impact due to the variation of all the parameters of the model using the bifurcation diagrams. In chaos theory, the small change in the initial condition and the parameters of the model can generate many consequences: we can recover chaotic dynamics, we can lose chaotic dynamics, we can recover hyperchaotic behavior, we can lose hyperchaotic behavior, we can get periodic orbit, and we can enter with 


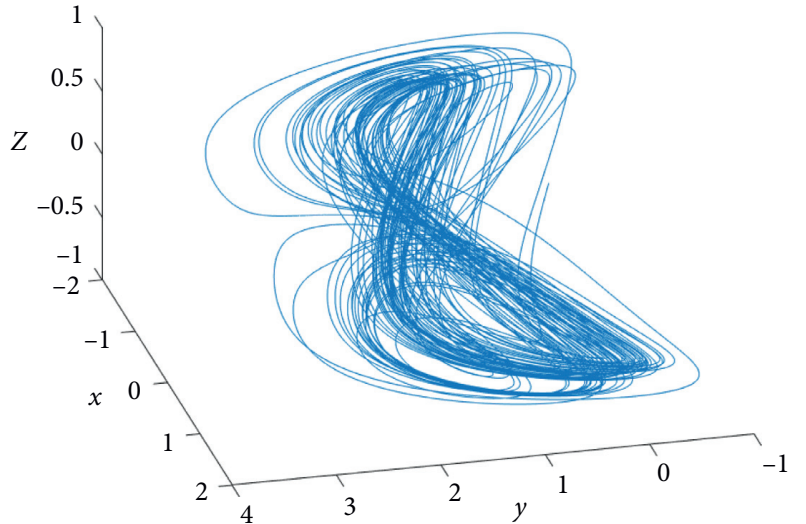

Figure 1: Interest rate, investment demand, and price exponent and with order $\alpha=0.95$.

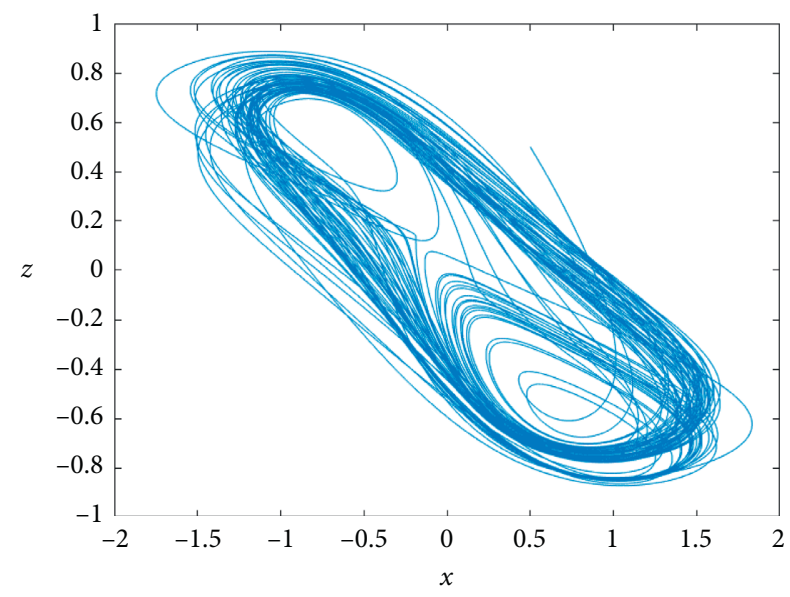

Figure 2: Interest rate and price exponent and with order $\alpha=0.95$.

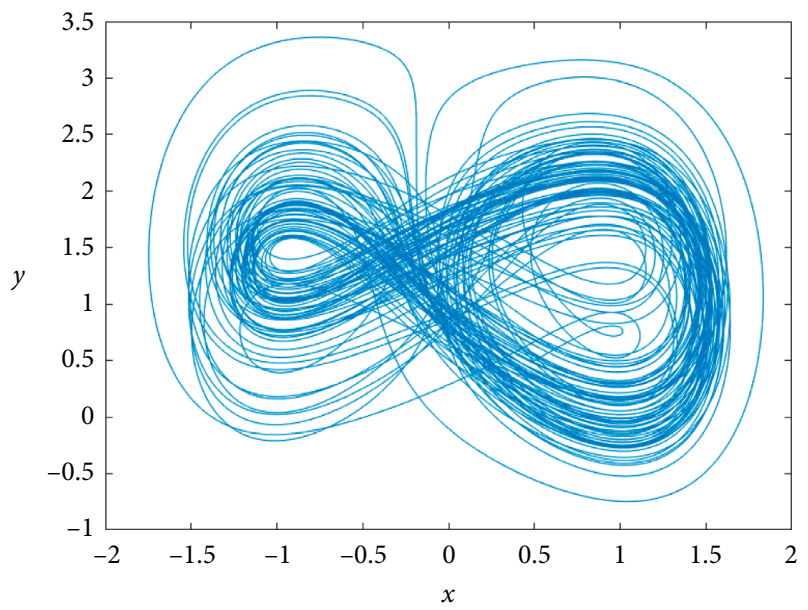

FIgURE 3: Interest rate and investment demand and with order $\alpha=0.95$.

period-doubling bifurcation, the primary system which the equilibrium point is not stable can become stable and many other properties which can be determined with bifurcation

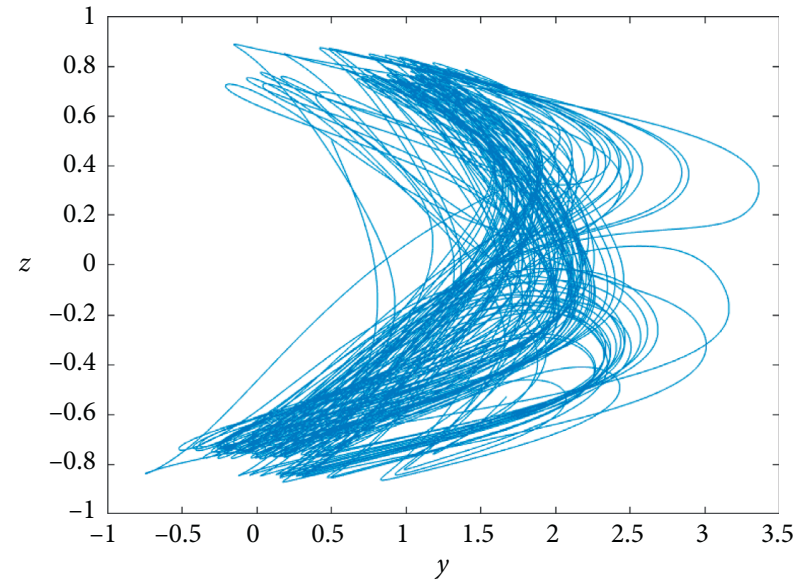

FIGURE 4: Investment demand and price exponent and with order $\alpha=0.95$.

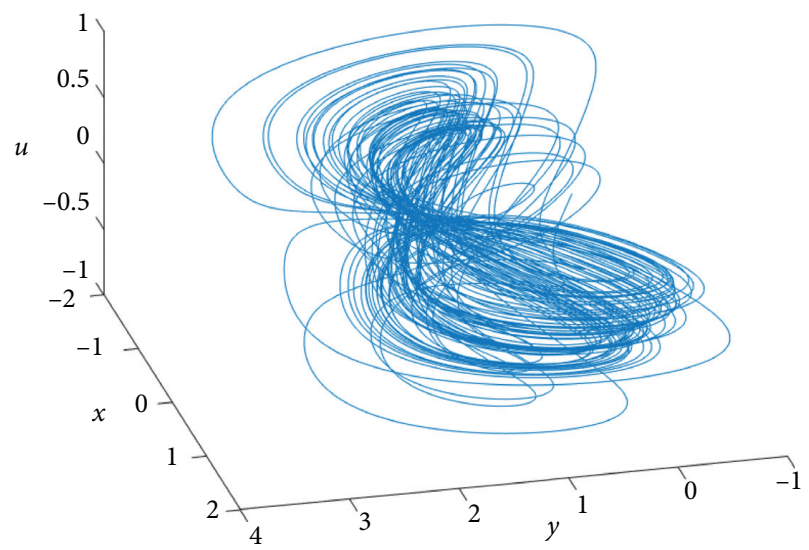

FIgURE 5: Interest rate, investment demand, and average profit margin and with order $\alpha=0.95$.

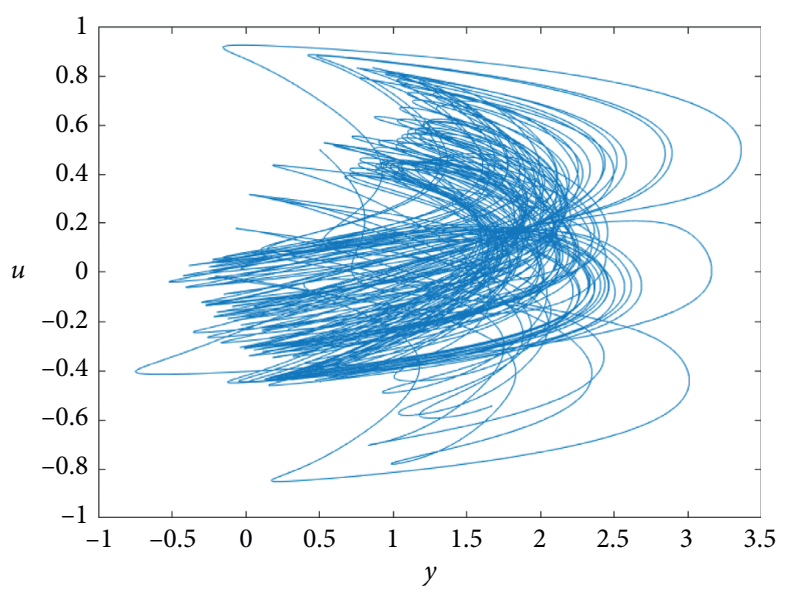

FIgURE 6: Investment demand and average profit margin and with order $\alpha=0.95$.

diagrams and the Lyapunov exponents. We also confirm the previous investigations by proving the existence of chaotic behaviors and hyperchaotic behaviors at the chosen 


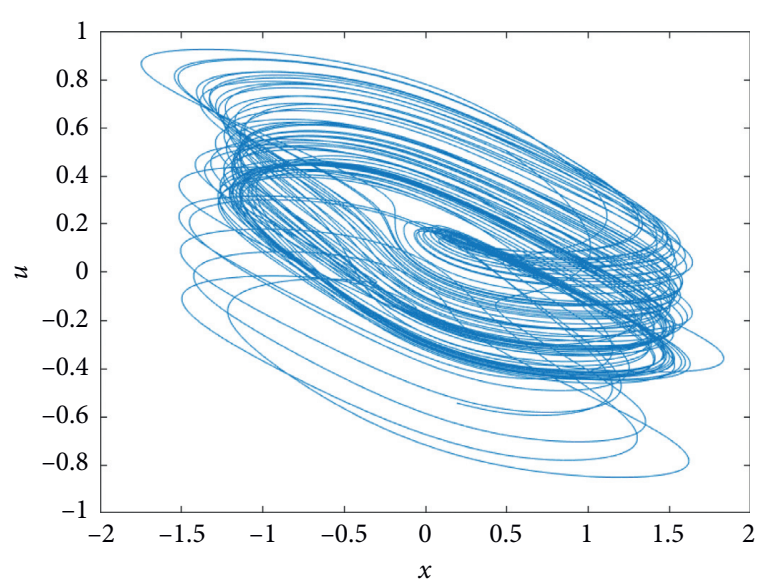

FIGURE 7: Interest rate and average profit margin and with order $\alpha=0.95$.

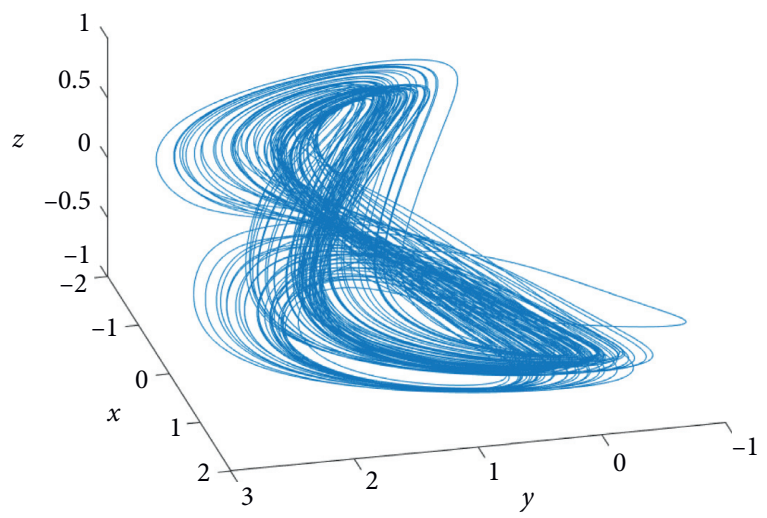

Figure 8: Interest rate, investment demand, and price exponent and with order $\alpha=0.90$.

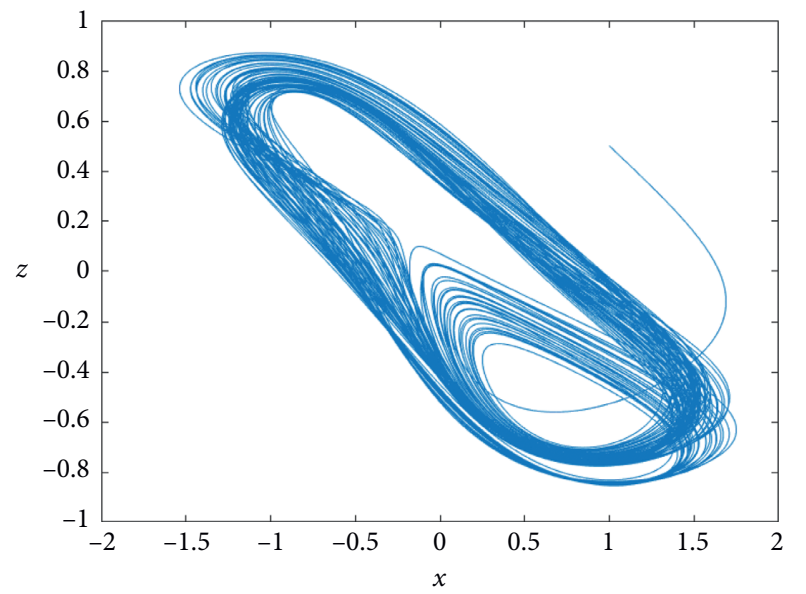

FIGURE 9: Interest rate and price exponent and with order $\alpha=0.90$.

fractional order $\alpha=0.95$. In this section, the analysis of the bifurcation diagrams and the Lyapunov exponents will be done according to the following investigations [9-15]. It is essential to mention that except for the last bifurcation

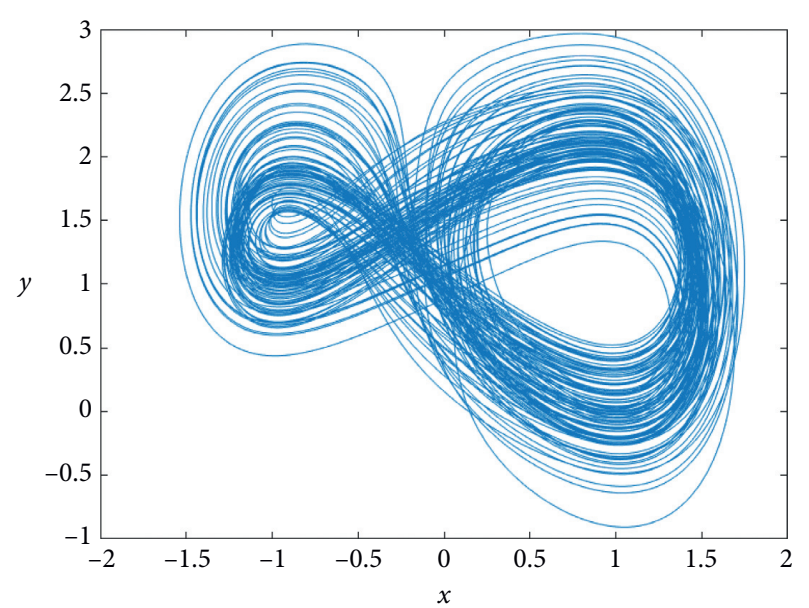

FIGURE 10: Interest rate and investment demand and with order $\alpha=0.90$.

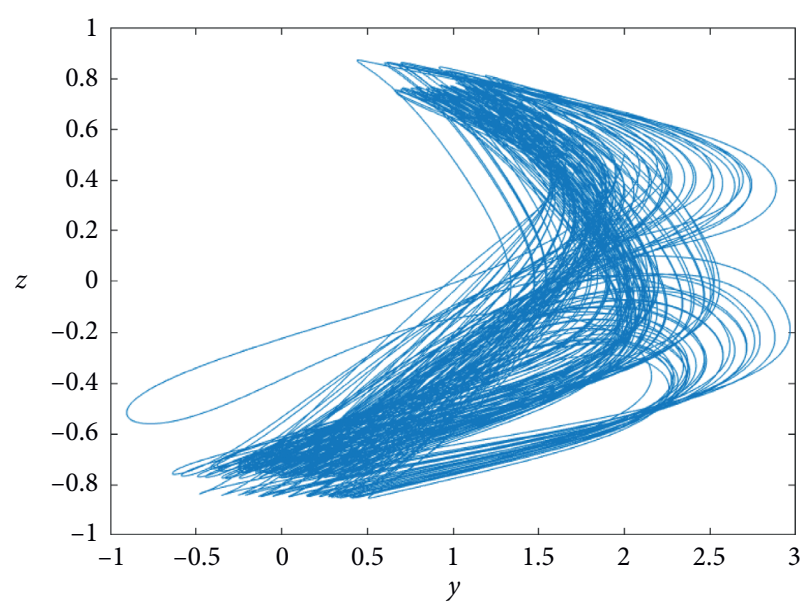

FIgURE 11: Investment demand and price exponent and with order $\alpha=0.90$.

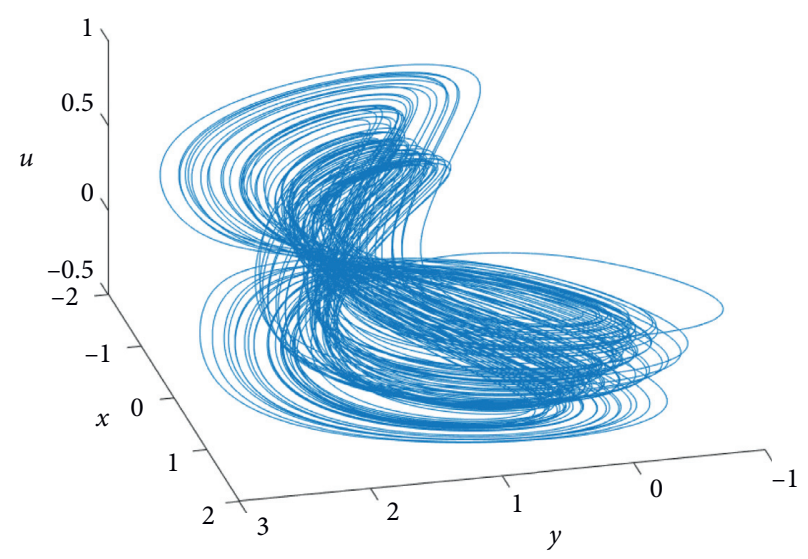

FiguRE 12: Interest rate, investment demand, and average profit margin and with order $\alpha=0.90$.

diagram, the considered step size to describe the variation of the parameters of the model is 0.001 . 


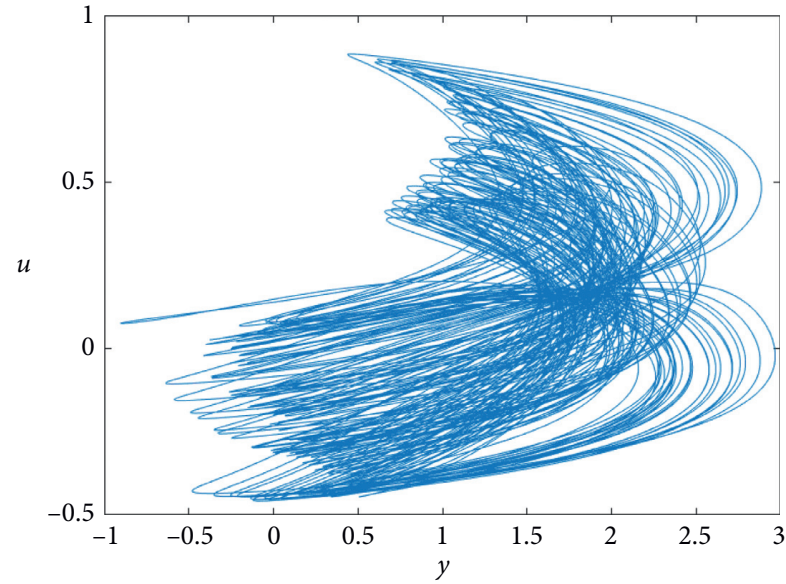

FIGURE 13: Investment demand and average profit margin and with order $\alpha=0.90$.

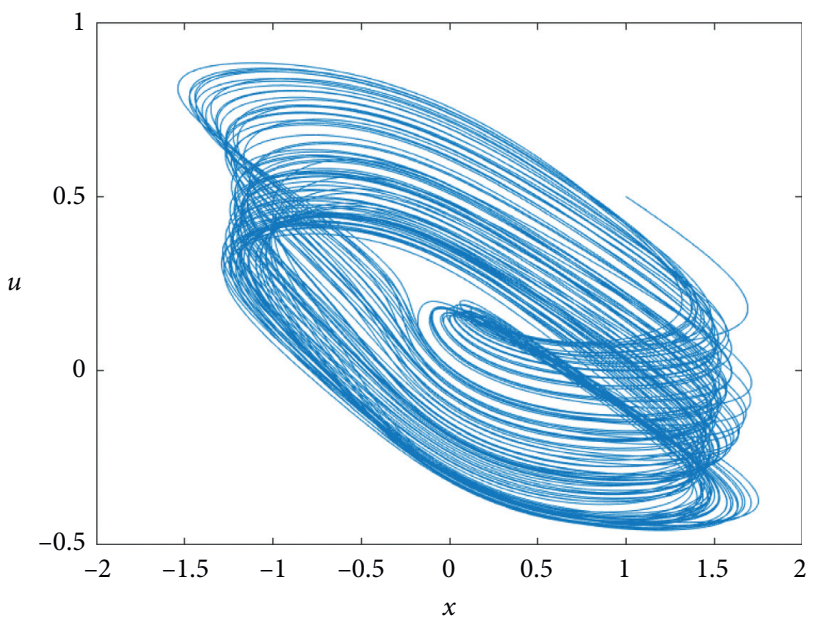

FIGURE 14: Interest rate and average profit margin and with order $\alpha=0.90$.

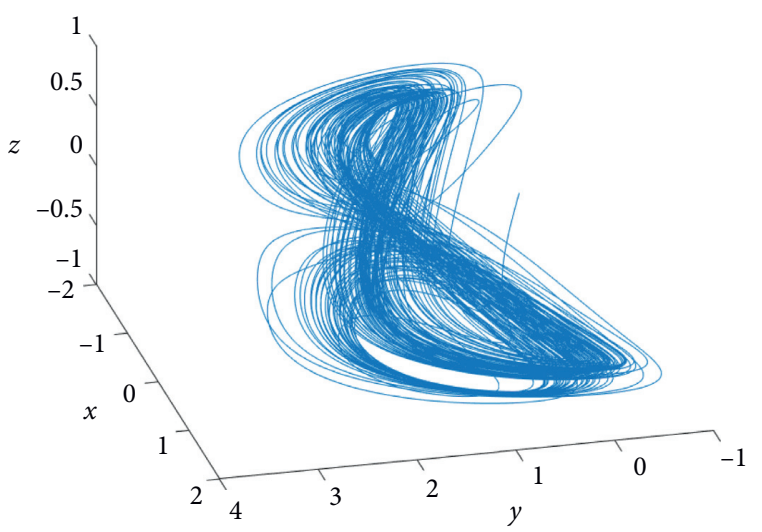

Figure 15: Interest rate, investment demand, and price exponent and with order $\alpha=0.88$.

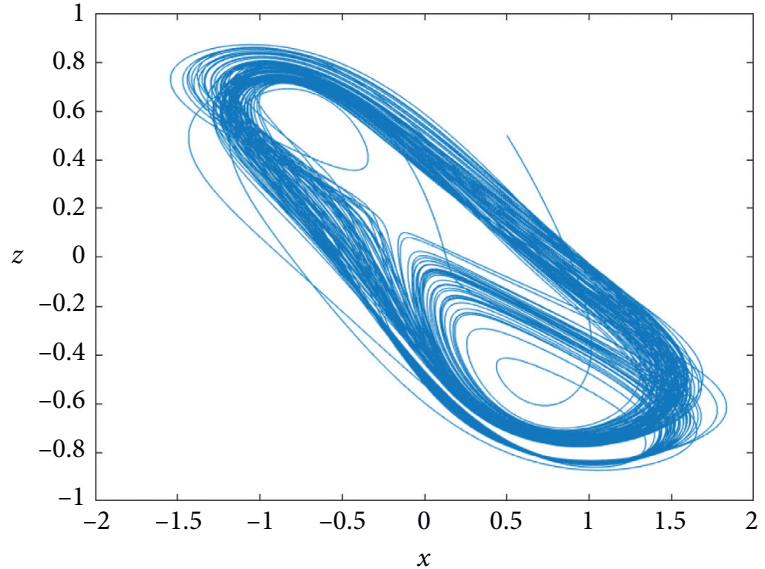

FIGURE 16: Interest rate and price exponent and with order $\alpha=0.88$.

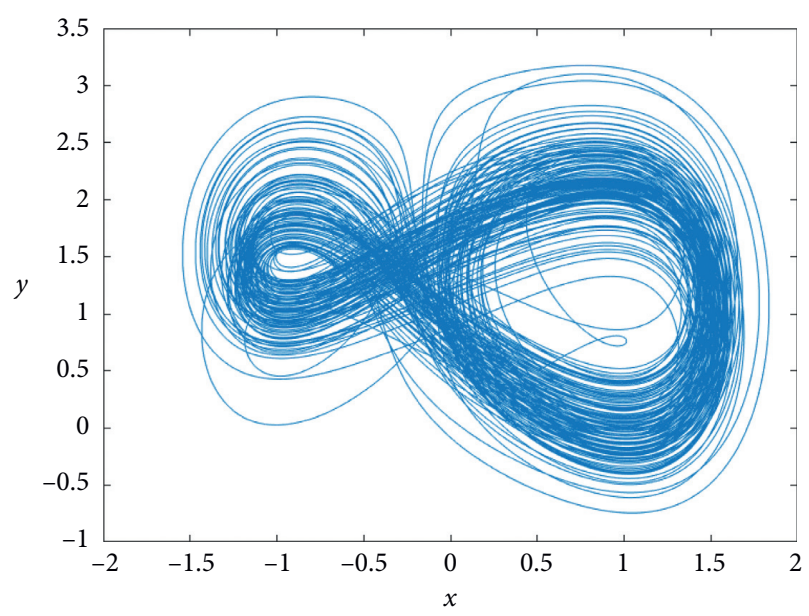

Figure 17: Interest rate and investment demand and with order $\alpha=0.88$.

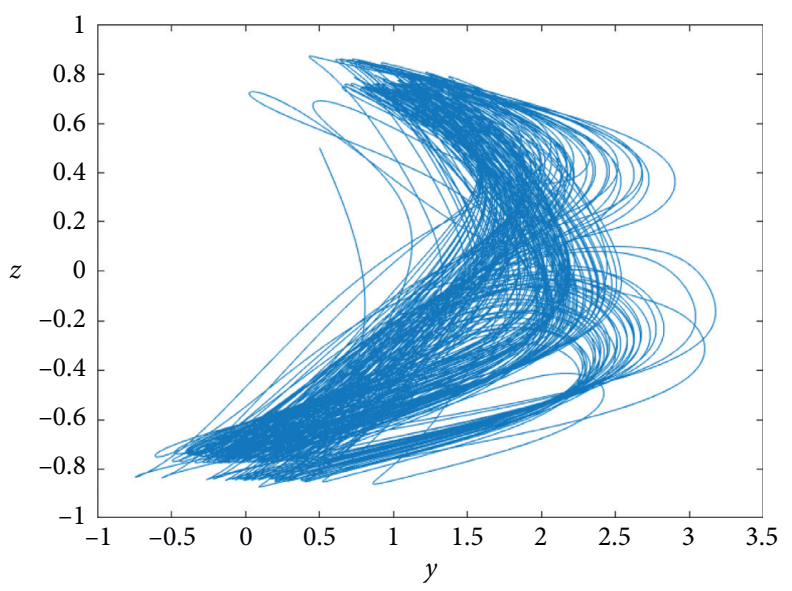

FIGURE 18: Investment demand and price exponent and with order $\alpha=0.88$. 


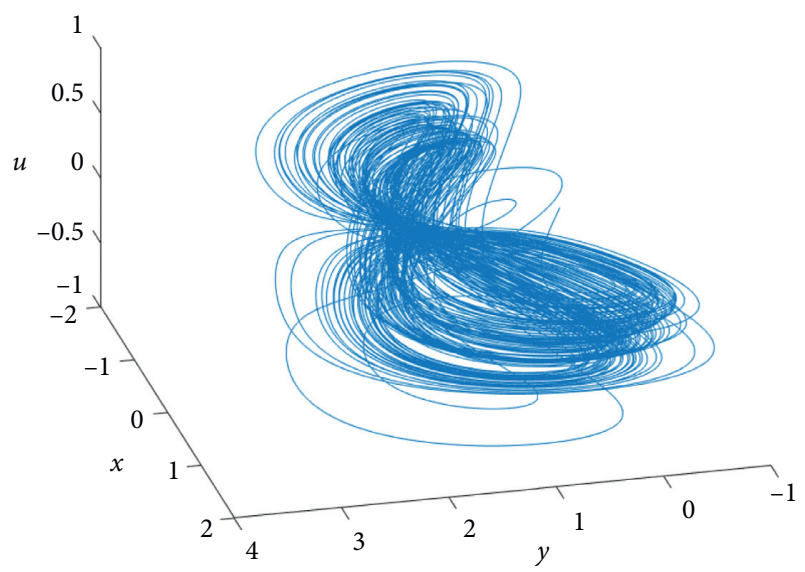

FIGURE 19: Interest rate, investment demand, and average profit margin and with order $\alpha=0.88$.

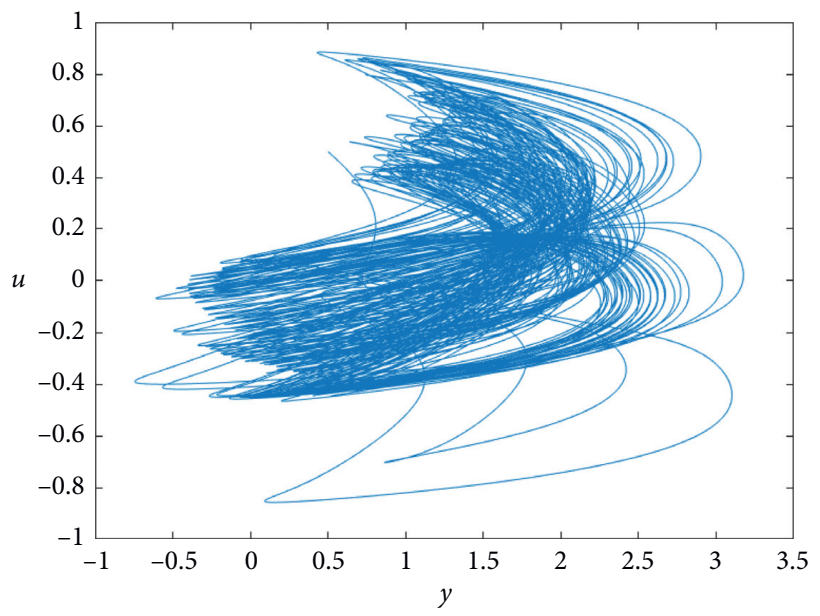

FIGURE 20: Investment demand and average profit margin and with order $\alpha=0.88$.

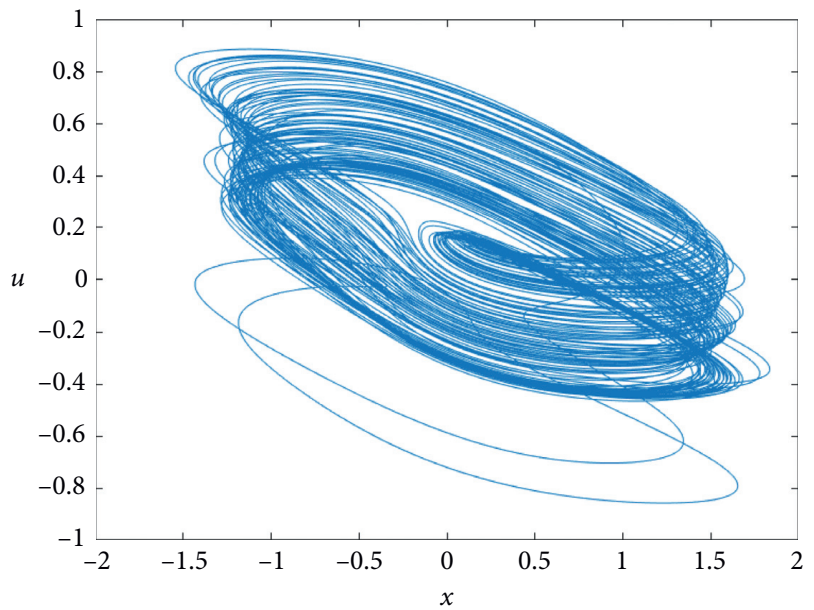

FIGURE 21: Interest rate and average profit margin and with order $\alpha=0.88$.

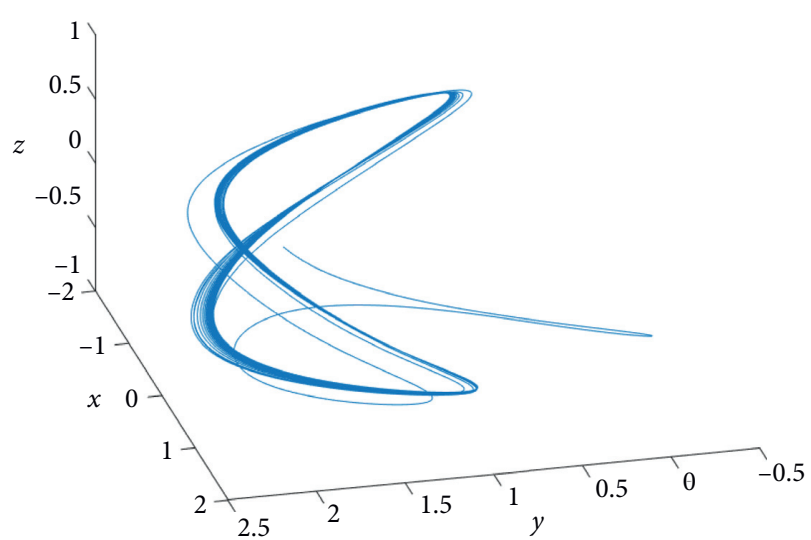

FIgURE 22: Interest rate, investment demand, and price exponent and with order $\alpha=0.45$.

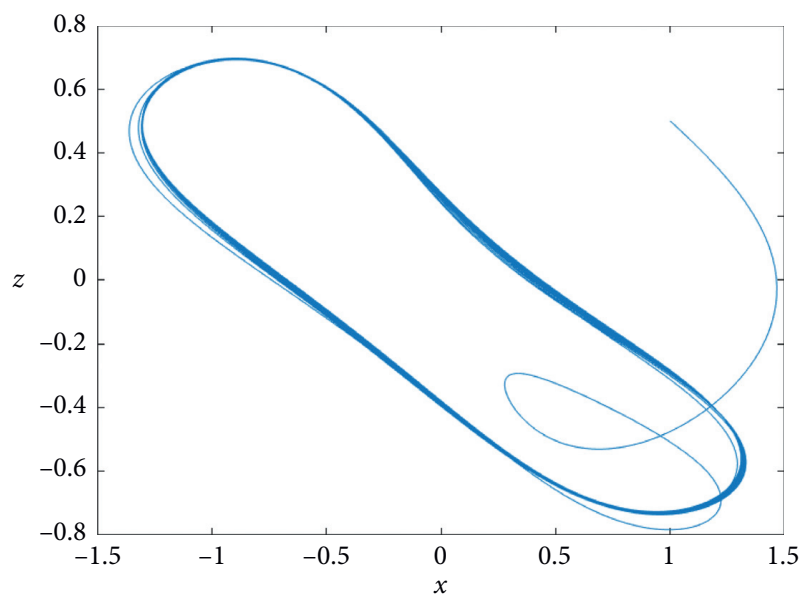

FIgURE 23: Interest rate and price exponent and with order $\alpha=0.45$.

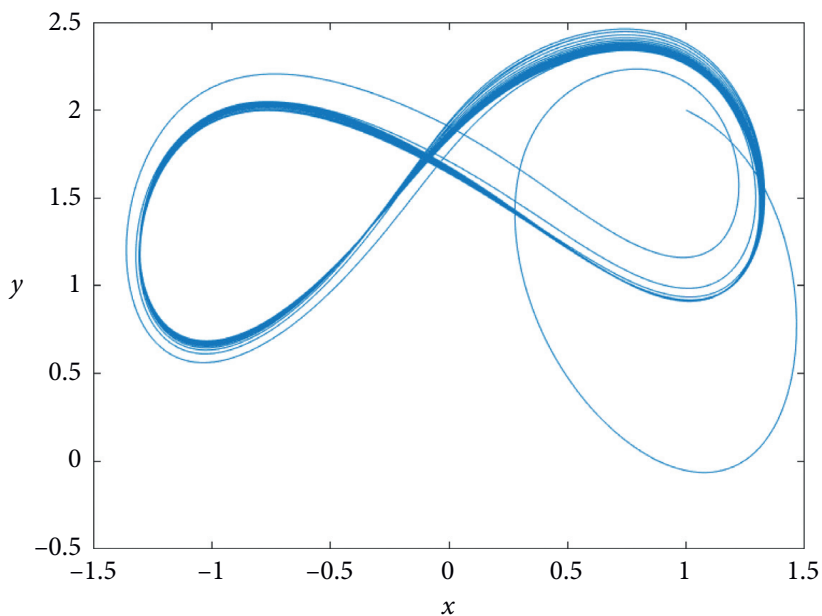

FIGURE 24: Interest rate and investment demand and with order $\alpha=0.45$. 


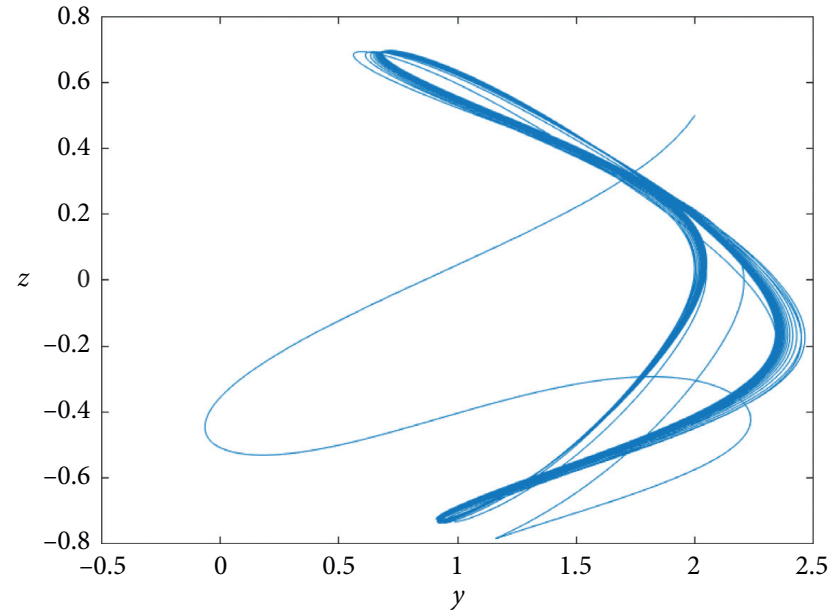

FIGURE 25: Investment demand and price exponent and with order $\alpha=0.45$.

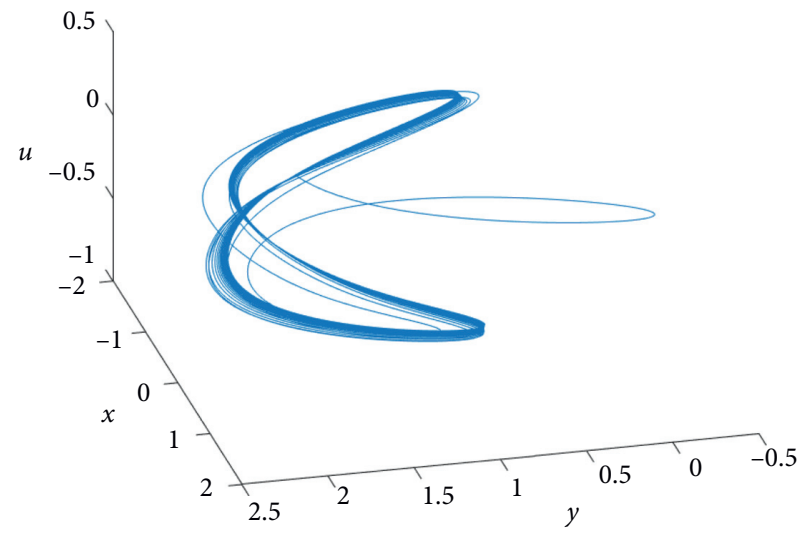

Figure 26: Interest rate, investment demand, and average profit margin and with order $\alpha=0.45$.

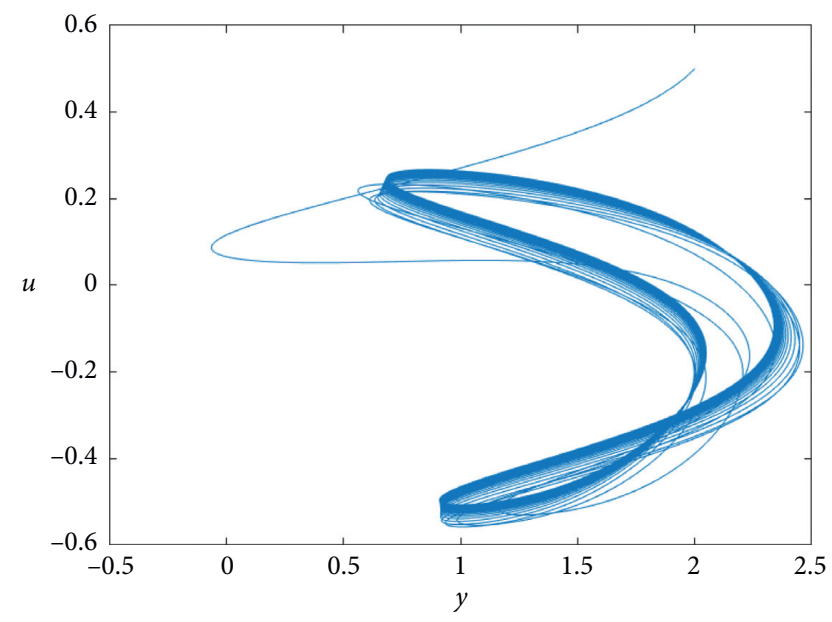

FIGURE 27: Investment demand and average profit margin and with order $\alpha=0.65$.

We begin by the variation of the saving rate $a$; we suppose the other parameters do not vary and are given by the per investment cost $b=0.2$, the elasticity of demands $c=1.2, d=0.2$, and the average profit margin influence $k=0.15$. In Figure 28 , we give the bifurcation diagram associated with the variation of the saving rate $a$ into the interval $(0,1)$. The motivations of the consideration of this interval are because, in practice, the saving rate can not belong outside of the interval $(0,1)$. When this case happens, the problem becomes irrealistic financial and economic viewpoints. For more visibility, we consider in our simulation the step size $h=0.001$. In Figure 28, the bifurcation diagram indicates for the order $\alpha=0.95$; when the saving rate increases, the system begins with hyperchaotic behaviors and continues with this dynamics behaviors as soon as the saving rate is into the interval $(0,0.775)$. But, in $(0.775,0.998)$, the system lost its hyperchaotic behaviors and became chaotic, and new hyperchaotic behaviors born in the neighborhood of 1 . For pieces of information which are not reported here, the system lost the hyperchaotic behaviors when the saving rate exceeds 1 and continues with perioddoubling bifurcation.

We continue with the variation of the per investment cost $b$ into $(0,1)$. The motivation of the choice of the interval involving the per investment cost is because this value in practice does not belong outside $(0,1)$. We fix the saving rate $a=0.5$, the elasticity of demands $c=1.2, d=0.2$, and the average profit margin influence $k=0.15$. We depict the bifurcation diagram according to the variation of per investment cost in Figure 29. We notice the system begins and continues with the hyperchaotic behaviors when the per investment cost $b$ is into $(0,0.3)$. When the per investment cost exceeds 0.3 , the system removes the hyperchaotic behaviors, and the system undergoes periodic orbit. We notice the behaviors such as the hyperchaotic, the chaotic, and the periodic orbit in our context depend on the choice of the per investment cost.

In the third step, the elasticity of demands $c$ varies into $(1,3)$. We fix saving rate $a=0.5$, the per investment cost $b=0.2, d=0.2$, and the average profit margin influence $k=0.15$. We depict the bifurcation diagram related to the variation of the elasticity of demands in Figure 30. The observations are summarized as follows. When the elasticity of demand is into $(1,2)$, the system generates hyperchaotic dynamics. In particular, in the interval $(1.5,2)$, we notice moderate hyperchaotic behaviors. In $(2,2.8)$, the system lost hyperchaotic behaviors, and the solutions of the system probably converge to the equilibrium points. Note that into this interval, the analysis is not trivial. Finally, when the elasticity of demands $c$ varies into $(2.8,3)$, new hyperchaotic behaviors start again.

In the last step, the average profit margin influence $k$ varies into $(0,1)$. In general, the presence of hyperchaotic behaviors is detected when $k$ varies into $(0,0.25)$ and $(0.7,1)$.

We finish by characterizing the presence of chaotic and hyperchaotic behaviors when the fractional order is used. The method used to calculate the Lyapunov exponents in fractional version can be found in [43]. We fix the conditions: the saving rate $a=0.5$, the per investment cost $b=0.2$, the elasticity of demands $c=1.2, d=0.2$, and the average 


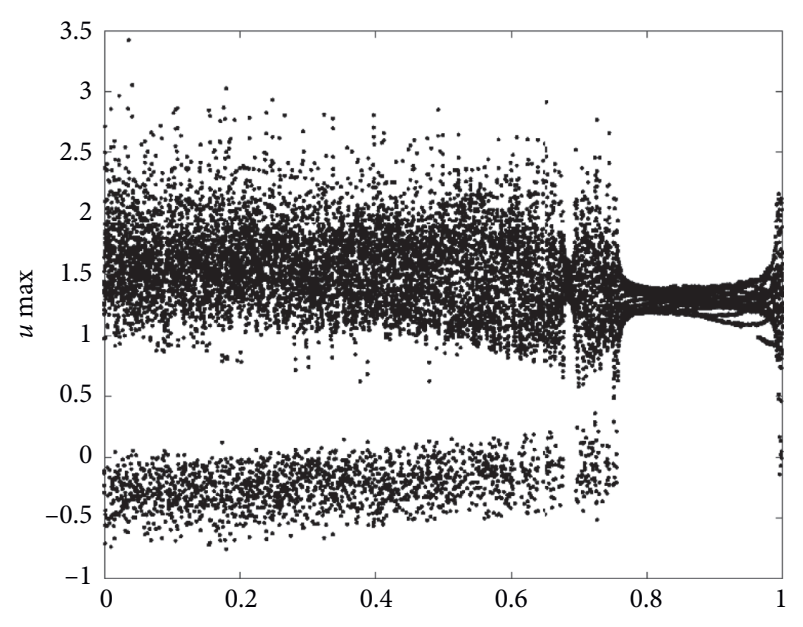

Figure 28: Bifurcation diagram $a$.

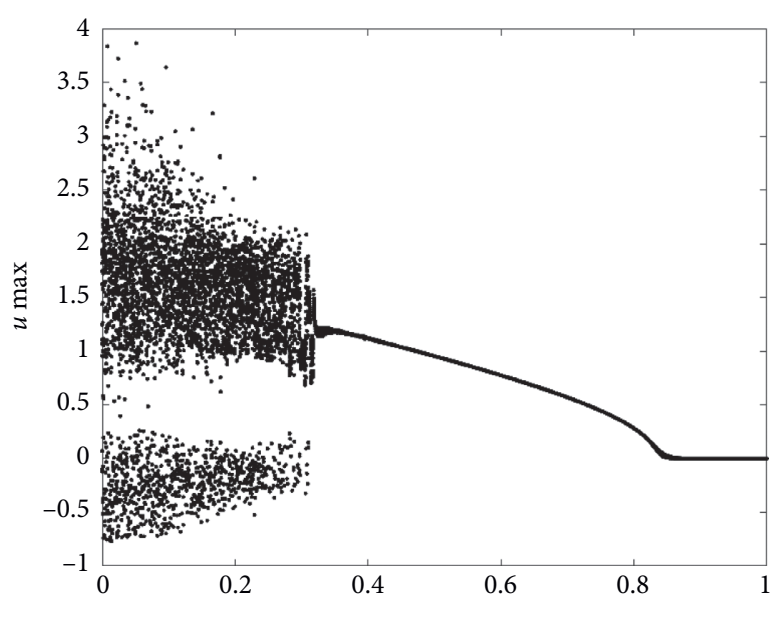

Figure 29: Bifurcation diagram $b$.

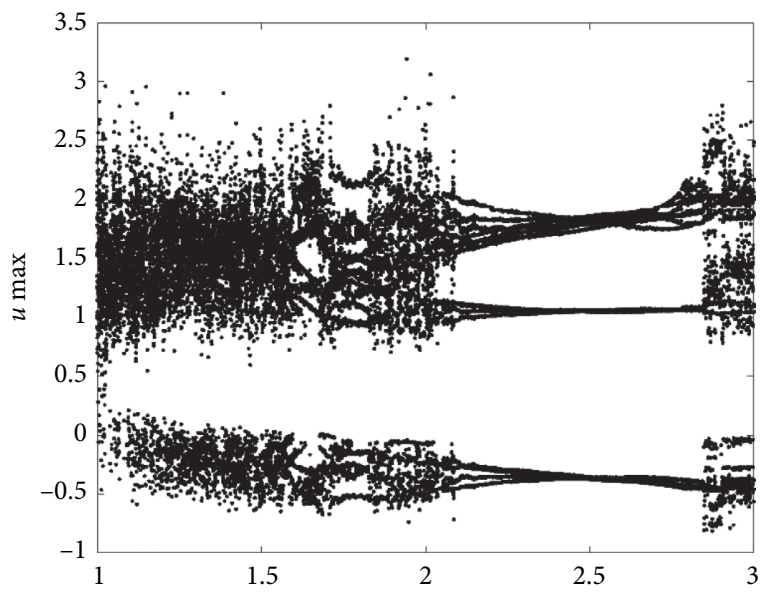

Figure 30: Bifurcation diagram $c$.

profit margin influence $k=0.15$. We maintain the initial conditions in Section 6. In our first analysis, we calculate the Lyapunov exponents for the different fractional-orders considered in the graphical representations. In other words, we will try to validate the hyperchaotic behaviors observed in the graphic representation sections. For $\alpha=0.45$, the Lyapunov exponents are given by the following:

$$
\begin{aligned}
& \mathrm{LE}_{1}=-0.0277, \\
& \mathrm{LE}_{2}=-0.9910, \\
& \mathrm{LE}_{3}=-0.7651, \\
& \mathrm{LE}_{4}=-8.2664 .
\end{aligned}
$$

Our first remark is systems (13)-(16) are dissipative because the sum of all Lyapunov exponents is negative. The absence of chaotic and hyperchaotic behaviors for the order $\alpha=0.45$ can be explained by the fact all the Lyapunov exponents are negative. Thus, for the order $\alpha=0.45$, we note the suppression of the hyperchaotic behaviors, which is explained by the negativity of all the Lyapunov exponents.

For $\alpha=0.88$, the Lyapunov exponents are given by the following numbers:

$$
\begin{aligned}
& \mathrm{LE}_{1}=0.2246, \\
& \mathrm{LE}_{2}=0.0284, \\
& \mathrm{LE}_{3}=-0.3535, \\
& \mathrm{LE}_{4}=-1.2650 .
\end{aligned}
$$

The dissipativity of the system is explained by the fact that the sum of all Lyapunov exponents is negative. We can observe two positive Lyapunov exponents; thus, this condition is necessary and sufficient for the detection of hyperchaotic behaviors. The fractional-order system (13)-(16) is hyperchaotic at order $\alpha=0.88$. Let mention that it is established by Danfa in [19]; in fractional-order context, the existence of two positive Lyapunov exponents is not an adequate definition. For example, in [19], the authors find some fractional-order hyperchaotic systems that have one positive Lyapunov exponent; we confirm this assumption in our investigations. For example, let the order $\alpha=0.90$, the Lyapunov exponents are as follows:

$$
\begin{aligned}
& \mathrm{LE}_{1}=0.2657, \\
& \mathrm{LE}_{2}=-0.0145, \\
& \mathrm{LE}_{3}=-0.2867, \\
& \mathrm{LE}_{4}=-1.1211 .
\end{aligned}
$$

We can observe our system is hyperchaotic, but strangely we get one positive Lyapunov exponent. For more pieces of information, see in [19]. The question related to the characterization of the nature of chaos in fractional context born, and note that the existence of one positive Lyapunov exponent becomes a necessary condition for the hyperchaotic behaviors but not sufficient. The alternative to supporting the hyperchaotic behaviors with the existence of one positive Lyapunov exponent is to combine it by the bifurcation diagrams. This question is an open problem in fractional calculus. 
For $\alpha=0.95$, the Lyapunov exponents are given by the following numbers:

$$
\begin{aligned}
& \mathrm{LE}_{1}=0.2113, \\
& \mathrm{LE}_{2}=0.0017, \\
& \mathrm{LE}_{3}=-0.3924, \\
& \mathrm{LE}_{4}=-0.8526 .
\end{aligned}
$$

With two positive $\mathrm{LE}_{1}$ and $\mathrm{LE}_{2}$, the hyperchaotic behavior is detected at $\alpha=0.95$, but the hyperchaotic behavior for our new hyperchaotic system is more complex at the order $\alpha=0.88$. The justification is the impact of the term $x^{4}$, and $\mathrm{LE}_{1}$ and $\mathrm{LE}_{2}$ are more significant at order $\alpha=0.88$.

To support the results in the bifurcation section, we describe in the following tables, the values of the Lyapunov exponents versus the small variation of the parameters of the model. We begin with the variation of the saving rate. Note that the Lyapunov exponent versus the variation of the parameter $a$ is represented in Table 1 . The considered order is $\alpha=0.95$.

We analyze with the aid of the bifurcation diagram. We notice the existence of one or two positive Lyapunov exponents into the interval $(0,0.7)$. Still, with the aid of its associated bifurcation diagram, we conclude the fractional system is hyperchaotic with a minimum one positive Lyapunov exponent. There exists one positive Lyapunov exponent when the parameter $\mathrm{a}$ is into the interval $(0.7,0.998)$. Furthermore, with the bifurcation diagram, we conclude our system is chaotic. It is indispensable to get the bifurcation diagram before concluding with one positive Lyapunov exponent in fractional context because we can find the order of the fractional derivative under which the presence of the hyperchaotic behaviors is detected with one positive Lyapunov exponent.

In Table 2, we consider the variation of the per investment cost $b$ into $(0,1)$. The Lyapunov exponents are assigned in the following table.

The results described in Table 2 are in good agreement with the analysis done in the bifurcation diagram related to the variation of the per investment cost $b$. We also notice two positive Lyapunov exponents into $[0,0.3)$, which confirm the hyperchaotic behaviors. Into interval $[0.3,1]$, when the per investment cost increases, it generates moderate hyperchaotic behavior and finish to remove the hyperchaotic behaviors into the dynamics of the system. All equilibrium points obtained into this interval for our system become automatically stable. It is normal because the chaos is removed.

In Table 3, we calculate the Lyapunov exponents according to the variation of the elasticity of demands $c$ into $(1,3)$. The same procedure, as described in the previous tables, is adopted. Here, due to the largest interval, we consider the variation of the elasticity of the demands $c$ into $(1,1.5)$.

We can observe two positive Lyapunov exponents at all line of Table 3, andwe can affirm the fractional-order system is hyperchaotic. Note that in this Table 3, we have no hyperchaotic behavior with one positive Lyapunov exponent. The values of the Lyapunov exponents are confirmed
TABLE 1: Lyapunov exponents versus $a$.

\begin{tabular}{lcccc}
\hline$a$ & $\mathrm{LE}_{1}$ & $\mathrm{LE}_{2}$ & $\mathrm{LE}_{3}$ & $\mathrm{LE}_{4}$ \\
\hline 0.0 & 0.0057 & -0.2680 & -0.3072 & -0.8495 \\
0.1 & 0.0401 & -0.2083 & -0.3305 & -0.8545 \\
0.2 & 0.0813 & -0.1548 & -0.3520 & -0.8581 \\
0.3 & 0.1271 & -0.1060 & -0.3701 & -0.8598 \\
0.4 & 0.1742 & -0.0579 & -0.3842 & -0.8585 \\
0.5 & 0.2113 & 0.0017 & -0.3924 & -0.8526 \\
0.6 & 0.2233 & 0.0866 & -0.3889 & -0.8404 \\
0.7 & 0.4597 & -0.0706 & -0.3692 & -0.8184 \\
0.8 & 0.4788 & -0.1037 & -0.3129 & -0.8025 \\
0.9 & 0.4678 & -0.1037 & -0.3056 & -0.7989 \\
0.995 & 0.4278 & -0.0743 & -0.3075 & -0.7989 \\
\hline
\end{tabular}

TABle 2: Lyapunov exponents versus $b$.

\begin{tabular}{lcccc}
\hline$b$ & $\mathrm{LE}_{1}$ & $\mathrm{LE}_{2}$ & $\mathrm{LE}_{3}$ & $\mathrm{LE}_{4}$ \\
\hline 0.0 & 0.2343 & 0.0183 & -0.2853 & -0.8646 \\
0.1 & 0.2193 & 0.0149 & -0.3362 & -0.8687 \\
0.2 & 0.2113 & 0.0017 & -0.3924 & -0.8526 \\
0.3 & 0.2619 & -0.1430 & -0.4236 & -0.8053 \\
0.4 & 0.1934 & -0.2965 & -0.4528 & -0.7678 \\
0.5 & 0.0927 & -0.3732 & -0.5441 & -0.7491 \\
0.6 & -0.0023 & -0.4360 & -0.6342 & -0.7381 \\
0.7 & -0.0796 & -0.4976 & -0.7201 & -0.7352 \\
0.8 & -0.1422 & -0.5577 & -0.7980 & -0.7432 \\
0.9 & -0.1942 & -0.6148 & -0.8646 & -0.7656 \\
1.0 & -0.2384 & -0.6674 & -0.9181 & -0.8041 \\
\hline
\end{tabular}

TABLE 3: Lyapunov exponents versus $c$.

\begin{tabular}{lcccc}
\hline$c$ & $\mathrm{LE}_{1}$ & $\mathrm{LE}_{2}$ & $\mathrm{LE}_{3}$ & $\mathrm{LE}_{4}$ \\
\hline 1.00 & 0.5564 & 0.0400 & -0.3685 & -0.7869 \\
1.10 & 0.2668 & 0.0585 & -0.3721 & -0.7458 \\
1.20 & 0.2113 & 0.0017 & -0.3924 & -0.8526 \\
1.30 & 0.1915 & 0.0067 & -0.3161 & -1.2144 \\
1.40 & 0.1343 & 0.0167 & -0.3287 & -1.3357 \\
1.50 & 0.0722 & 0.0356 & -0.3459 & -1.4590 \\
\hline
\end{tabular}

by the bifurcation diagrams related to the variation of the parameter $c$.

The main contribution of this section shows the sign of the Lyapunov exponent is not sufficient to characterize the hyperchaotic dynamics. By our investigation, we can remark as Danca; there exist hyperchaotic systems with one positive Lyapunov exponent. In conclusion, to characterize the hyperchaotic system, it necessaries to confirm the work with the bifurcations diagrams. The Lyapunov exponent is known strong sensible to the initial condition. In the context of fractional-order derivative, the Lyapunov exponents depend on the initial conditions and the values of the Caputo derivative.

\section{Stability Analysis}

In this section, we study the local stability of the equilibrium points. We mainly use the so-called Matignon criterion [44] because, in fractional calculus, the negativity of the real part 
of the eigenvalues of the Jacobian matrix is not sufficient for the local stability. Let us describe the procedure which we will adopt in this section. There exist nowadays two methods to evaluate the stability of our related problem. The first is to use the Laplace transformation and the second is to use the Matignon criterion. The Matignon criterion is algebraic. The first step consists of determining the equilibrium points of the studied system. The second step consists of evaluating the Jacobian matrix at the equilibrium points. The third condition consists of determining the eigenvalues of the considered Jacobian matrix. In addition, as the last condition, we should verify if all the eigenvalues satisfy the socalled Mategnon criterion expressed as the form

$$
\left|\arg \left(\lambda_{i}\left(J_{\text {equi }}\right)\right)\right|=\pi>\alpha \pi / 2,
$$

where $i=1,2, \ldots, n$ and $\lambda_{i}\left(J_{\text {equi }}\right)$ represent the eigenvalue of the Jacobian matrix evaluated at the equilibrium point. Equation (110) is the main difference between the stability with integer-order derivative and the stability with fractional-order derivative. It is essential to mention that if all the eigenvalues of the Jacobian matrix satisfy the condition (110), we get the local asymptotic stability. The present procedure does not give global stability, which needs to find suitable Lyapunov function. The global stability is not trivial in general for the hyperchaotic model because many of its equilibrium points are not stable. First of all, we determine the equilibrium points, and after calculations, we obtain the following relationships:

$$
\begin{aligned}
& x_{1}^{*}=-\left[\frac{c k-c d-a b c k-b k}{c k-c d}\right]^{1 / 4}, \\
& x_{2}^{*}=\left[\frac{c k-c d-a b c k-b k}{c k-c d}\right]^{1 / 4}, \\
& y^{*}=\frac{a c k+k}{c k-c d} \\
& z_{1}^{*}=\frac{x_{1}^{*}}{c}, \\
& z_{2}^{*}=\frac{x_{2}^{*}}{c}, \\
& u_{1}^{*}=-\frac{d x_{1}^{*} y^{*}}{k}, \\
& u_{2}^{*}=-\frac{d x_{2}^{*} y^{*}}{k} .
\end{aligned}
$$

Using the values of the saving rate $a=0.5$, the per investment cost $b=0.2$, the elasticity of demands $c=1.2$, $d=0.2$, and the average profit margin influence $k=0.15$, the equilibrium points are given by $E_{q_{1}}=(0,5,0,0)$, $E_{q_{2}}=(-0.158,-4,-0.132,-0.842), \quad$ and $E_{q_{3}}^{q_{2}}=(0.158,-4,0.132,0.842)$. The Jacobian matrix which is also used to determine the Lyapunov exponent is given by the following matrix:

$$
J=\left(\begin{array}{cccc}
y-a & x & 1 & 1 \\
-4 x^{3} & -b & 0 & 0 \\
-1 & 0 & -c & 0 \\
-d y & -d x & 0 & -k
\end{array}\right)
$$

We begin our local stability study by the equilibrium point given by $E_{q_{1}}=(0,5,0,0)$ according to the Matignon criterion previously described. Here, the values of the parameter are used directly in the calculations. The following matrix is the Jacobian matrix at the point $E_{q_{1}}$ :

$$
J_{E_{q_{1}}}=\left(\begin{array}{cccc}
4.5 & 0 & 1 & 1 \\
0 & -0.2 & 0 & 0 \\
-1 & 0 & -1.2 & 0 \\
-1 & 0 & 0 & -0.15
\end{array}\right) .
$$

Using Matlab, the eigenvalues of the Jacobian matrix at the point $E_{q_{1}}$ is given by the following values $\lambda_{1}=4.093, \lambda_{2}=-0.230, \lambda_{3}=0.106$, and $\lambda_{4}=-0.2$. By the Matignon criterion, we have that $\left|\arg \left(\lambda_{1}\right)\right|=0<\alpha \pi / 2$, $\left|\arg \left(\lambda_{2}\right)\right|=\pi>\alpha \pi / 2, \quad\left|\arg \left(\lambda_{3}\right)\right|=0<\alpha \pi / 2, \quad$ and $\left|\arg \left(\lambda_{4}\right)\right|=\pi>\alpha \pi / 2$. Thus, the equilibrium point $E_{q_{1}}$ is not stable because all the eigenvalues do not satisfy the Matignon criterion given at equation (110).

We continue our local stability study by the equilibrium point given by $E_{q_{2}}=(-0.158,-4,-0.132,-0.842)$ according to the Matignon criterion. As recalled in the previous point, here, the values of the parameters are used directly in the calculations. The following matrix is the Jacobian matrix at the point $E_{q_{2}}$ :

$$
J_{E_{q 2}}=\left(\begin{array}{cccc}
-4.5 & -0.158 & 1 & 1 \\
0.0158 & -0.2 & 0 & 0 \\
-1 & 0 & -1.2 & 0 \\
0.8 & 0.0316 & 0 & -0.15
\end{array}\right) .
$$

The Jacobian matrix evaluated at the points $E_{q_{2}}$ has the following eigenvalues $\lambda_{1}=4.4840, \lambda_{2}=-0.912, \lambda_{3}=0.771$, and $\lambda_{4}=-0.199$. By the Matignon criterion, we have that $\left|\arg \left(\lambda_{1}\right)\right|=0<\alpha \pi / 2, \quad\left|\arg \left(\lambda_{2}\right)\right|=\pi>\alpha \pi / 2$, $\left|\arg \left(\lambda_{3}\right)\right|=0<\alpha \pi / 2$, and $\left|\arg \left(\lambda_{4}\right)\right|=\pi>\alpha \pi / 2$. Thus, the equilibrium point $E_{q_{2}}$ is also not stable.

We finish our local stability study by the equilibrium point given by $E_{q_{3}}=(0.158,-4,0.132,0.842)$ according to the Matignon criterion. We suppose the same assumptions related to the values of the parameters of the model. The following matrix is the Jacobian matrix at the point $E_{q 3}$ :

$$
J_{E_{q 2}}=\left(\begin{array}{cccc}
-4.5 & 0.158 & 1 & 1 \\
-0.0158 & -0.2 & 0 & 0 \\
-1 & 0 & -1.2 & 0 \\
0.8 & -0.0316 & 0 & -0.15
\end{array}\right) .
$$


The Jacobian matrix evaluated at the points $E_{q 3}$ has the following eigenvalues $\lambda_{1}=4.4840, \lambda_{2}=-0.912, \lambda_{3}=0.771$, and $\lambda_{4}=-0.199$. By the Matignon criterion, we have that $\left|\arg \left(\lambda_{1}\right)\right|=0<\alpha \pi / 2, \quad\left|\arg \left(\lambda_{2}\right)\right|=\pi>\alpha \pi / 2$, $\left|\arg \left(\lambda_{3}\right)\right|=0<\alpha \pi / 2$, and $\left|\arg \left(\lambda_{4}\right)\right|=\pi>\alpha \pi / 2$. Thus, the equilibrium point $E_{q_{3}}$ is also not stable. Finally, we conclude all the equilibrium points are not stable.

\section{Conclusion}

In this paper, we have focused on the fractional $4 \mathrm{D}$ hyperchaotic financial model in the context of the fractional calculus. We have mainly focused on the existence and the uniqueness of the solutions of the fractional 4D hyperchaotic financial model described by the generalized Caputo-Liouville derivative. We also proposed a novel numerical scheme based on the fractional integral for getting the approximate solutions of the hyperchaotic financial model. The results are validated by the bifurcation, the Lyapunov exponent, and the stability analysis. The solutions are represented graphically to observe the behavior of the solutions. Significant results appear in this paper such as the impact of the fractional-order derivative in the hyperchaotic behaviors, the effect of the variations of the saving rate, the average profit margin influence, the elasticity of demands, and the variation of the per investment cost in the dynamics of the proposed model. It is noticed they can generate hyperchaotic and chaotic behaviors when their values change in time. The proposed numerical scheme has many advantages due to the fact the stability and the convergence of the numerical method come from the Lipschitz continuous of the drift functions of the model. Another significant result is the stability of the equilibrium point depends strongly on the value of the used fractional-order derivative. For future works, the problem related to adaptative control of this new chaotic model can be investigated. We can observe in many financial models with fractional-order derivative; the incommensurable model is better to describe the concrete market behaviors; therefore, in the future direction of the investigations, we will investigate the phase portrait, the bifurcation, and the stability of the $4 \mathrm{D}$ financial hyperchaotic model with Caputo derivatives with different orders. This problem is opened in the literature and constitutes a new challenge for researchers in this field.

\section{Data Availability}

No data were used to support this study.

\section{Conflicts of Interest}

The authors declare that they have no conflicts of interest.

\section{References}

[1] X. Baogui and L. Yuting, "0-1 Test for Chaos in a fractional order financial System with investment incentive," Abstract and Applied Analysis, vol. 10, Article ID 876298, 2013.
[2] E. N. Lorenz, "Deterministic nonperiodic flow," Journal of the Atmospheric Sciences, vol. 20, no. 2, pp. 130-141, 1963.

[3] Y. Xu and Z. He, "Synchronization of variable order fractional financial system via active control method," Central European Journal of Physics, vol. 11, no. 6, 2013.

[4] Q. Gao and J. Ma, "Chaos and Hopf bifurcation of a finance system," Nonlinear Dynamics, vol. 58, no. 1-2, pp. 209-216, 2009.

[5] Z. Wang, X. Huang, and G. Shi, "Analysis of nonlinear dynamics and chaos in a fractional order financial system with time delay," Computers \& Mathematics with Applications, vol. 62, no. 3, pp. 1531-1539, 2011.

[6] C. Chen, T. Fan, and B. Wang, "Inverse optimal control of hyperchaotic finance system," World Journal of Modelling and Simulation, vol. 10, no. 2, pp. 83-91, 2014.

[7] H. Yu, G. Cai, and Y. Li, "Dynamic analysis and control of a new hyperchaotic finance system," Nonlinear Dynamics, vol. 67, no. 3, pp. 2171-2182, 2012.

[8] D. Kumar and S. Kumar, "Construction of four dimensional chaotic finance model and its applications," International Journal of Pure and Applied Mathematics, vol. 118, no. 22, pp. 1171-1187, 2018.

[9] S. He, K. Sun, X. Mei, B. Yan, and S. Xu, "Numerical analysis of a fractional-order chaotic system based on conformable fractional-order derivative," European Physical Journal Plus, vol. 132, p. 36, 2017.

[10] S. He, K. Sun, H. Wang, X. Mei, and Y. Sun, "Generalized synchronization of fractional-order hyperchaotic systems and its DSP implementation," Nonlinear Dynamics, vol. 92, no. 1, pp. 85-96, 2018.

[11] Ch. Zhou, Z. Li, Y. Zeng, and S. Zhang, "A novel 3D fractional-order chaotic system with," International Journal of Bifurcation and Chaos, vol. 29, no. 1, Article ID 1950004, 2019.

[12] S. Zhang, Y. Zeng, Z. Li, and C. Zhou, "Hidden extreme multistability, antimonotonicity and offset boosting control in a novel fractional-order hyperchaotic system without equilibrium," International Journal of Bifurcation and Chaos, vol. 28, no. 13, Article ID 1850167, 2018.

[13] V.-T. Pham, C. Volos, S. Jafari, and T. Kapitaniak, "Coexistence of hidden chaotic attractors in a novel no-equilibrium system," Nonlinear Dynamics, vol. 87, no. 3, pp. 2001-2010, 2017.

[14] V.-T. Pham, S. Vaidyanathan, C. Volos, S. Jafari, and S. T. Kingni, "A no-equilibrium hyperchaotic system with a cubic nonlinear term," Optik, vol. 127, no. 6, pp. 3259-3265, 2016.

[15] M. Shirkavand and M. Pourgholi, "Robust fixed-time synchronization of fractional order chaotic using free chattering nonsingular adaptive fractional sliding mode controller design," Chaos, Solitons \& Fractals, vol. 113, pp. 135-147, 2018.

[16] K. M. Owolabi, "Numerical analysis and pattern formation process for space fractional superdiffusive systems," Discrete \& Continuous Dynamical Systems Series S, vol. 12, no. 3, pp. 543-566, 2019.

[17] N. Sene, "Stokes' first problem for heated flat plate with Atangana-Baleanu fractional derivative," Chaos, Solitons \& Fractals, vol. 117, pp. 68-75, 2018.

[18] M. Yavuz and N. Sene, "Approximate solutions of the model describing fluid flow using generalized $\rho$-Laplace transform method and heat balance integral method," Axioms, vol. 9, no. 4, p. 123, 2020.

[19] M.-F. Danca, "Lyapunov exponents of a discontinuous 4D hyperchaotic system of integer or fractional order," Entropy, vol. 20, no. 5, p. 337, 2018. 
[20] A. Atangana, E. Bonyah, and A. A. Elsadany, "A fractional order optimal 4D financial model with Mittag-Leffler law," Chiness Journal of Physics, vol. 65, pp. 38-53, 2020.

[21] M. A. Khan, "The dynamics of the new chaotic system through the Caputo-Fabrizio and Atangana-Baleanu fractional operators," Advances in Mechanical Engineering, vol. 11, no. 7, pp. 1-12, 2019.

[22] T. Mekkaoui, Z. Hammouch, D. Kumar, and J. Singh, "A new approximation scheme for solving ordinary differential equation with Gomez-atangana-caputo fractional derivative," Methods of Mathematical Modelling, vol. 51, p. 12, 2019.

[23] M. Diouf and N. Sene, "Analysis of the financial chaotic model with the fractional derivative operator," Complexity, vol. 2020, Article ID 9845031, , 2020.

[24] K. Ali Abro, "A fractional and analytic investigation of thermo-diffusion process on free convection flow: an application to surface modification technology," European Physical Journal Plus, vol. 135, no. 1, pp. 31-41, 2019.

[25] K. Saad, D. Baleanu, and A. Atangana, "New fractional derivatives applied to the Korteweg-de Vries and Korteweg-de Vries-Burger's equations," Computational and Applied Mathematics, vol. 37, no. 6, 2018.

[26] N. Sene, "Global asymptotic stability of the fractional differential equations," Journal of Nonlinear Sciences and Applications, vol. 13, pp. 171-175, 2019.

[27] K. A. Abro, A. Siyal, and A. Atangana, "Thermal stratification of rotational second-grade fluid through fractional differential operators," Journal of Thermal Analysis and Calorimetry, 2020.

[28] N. Sene and A. Atangana, "Integral-balance methods for the fractional diffusion equation described by the caputo-generalized fractional derivative," Methods of Mathematical Modelling, vol. 83, p. 22, 2019.

[29] M. Yavuz, "Characterizations of two different fractional operators without singular kernel," Mathematical Modelling of Natural Phenomena, vol. 14, no. 3, p. 302, 2019.

[30] K. Ali Abro and A. Atangana, "Mathematical analysis of memristor through fractal-fractional differential operators: a numerical study," Mathematical Methods in the Applied Sciences, vol. 43, no. 10, pp. 6378-6395, 2020.

[31] K. Ali Abro and A. Atangana, "Role of non-integer and integer order differentiations on the relaxation phenomena of viscoelastic fluid," Physica Scripta, vol. 95, Article ID 035228, 2020.

[32] N. Sene and A. N. Fall, "Homotopy perturbation $\rho$-Laplace transform method and its application to the fractional diffusion equation and the fractional diffusion-reaction equation," Fractal and Fractional, vol. 3, no. 2, p. 14, 2019.

[33] N. Sene, "Integral balance methods for stokes' first, equation described by the left generalized fractional derivative," Physics, vol. 1, pp. 154-166, 2019.

[34] N. Sene, "Second-grade fluid model with Caputo-Liouville generalized fractional derivative," Chaos, Solitons \& Fractals, vol. 133, Article ID 109631, 2020.

[35] A. A. Kilbas, H. M. Srivastava, and J. J. Trujillo, Theory and Applications of Fractional Differential Equations, NorthHolland Mathematics Studies, Elsevier, Amsterdam, The Netherlands, 2006.

[36] I. Podlubny, "Fractional differential equations," Mathematics in Science and Engineering, Academic Press, New York, NY, USA, 1999.

[37] J. Fahd and T. Abdeljawad, "A modified Laplace transform for certain generalized fractional operators," Nonlinear Analysis, vol. 2, pp. 88-98, 2018.
[38] N. Sene, "Analytical solutions and numerical schemes of certain generalized fractional diffusion models," European Physical Journal Plus, vol. 134, p. 199, 2019.

[39] N. Sene, "Stability analysis of the generalized fractional differential equations with and without exogenous inputs," Journal of Nonlinear Sciences and Applications, vol. 12, no. 9, pp. 562-572, 2019.

[40] J. Fahd, T. Abdeljawad, and D. Baleanu, "On the generalized fractional derivatives and their Caputo modification," Journal of Nonlinear Sciences and Applications, vol. 10, pp. 2607-2619, 2017.

[41] M. Yavuz and N. Sene, "Stability analysis and numerical computation of the fractional Predator-Prey model with the harvesting rate," Fractal and Fractional, vol. 4, no. 3, p. 35, 2020.

[42] R. Garrappa, "Numerical solution of fractional differential equations: a survey and a software tutorial," Mathematics, vol. 6, no. 2, p. 16, 2018.

[43] M.-F. Danca and N. Kuznetsov, "Matlab code for Lyapunov exponents of fractional-order systems," International Journal of Bifurcation and Chaos, vol. 28, no. 5, Article ID 1850067, 2018.

[44] D. Matignon, "Stability results on fractional differential equations to control processing," in Proceedings of the Computational Engineering in Syatems and Application Multiconference; IMACS, pp. 963-968, Lille, France, July 1996. 